\title{
Generic Neighbor Discovery Accelerations in Mobile Applications
}

\author{
DESHENG ZHANG and TIAN HE, University of Minnesota \\ YUNHUAI LIU, Third Research Institute of Ministry of Public Security, China \\ YU GU, IBM Research at Austin \\ FAN YE, Stony Brook University \\ RAGHU K. GANTI and HUI LEI, IBM T. J. Watson Research Center
}

\begin{abstract}
As a supporting primitive of many mobile applications, neighbor discovery identifies nearby devices so that they can exchange information and collaborate in a peer-to-peer manner. To date, discovery schemes trade a long latency for energy efficiency and require a collaborative duty cycle pattern, and thus they are not suitable for interactive mobile applications where a user is unable to configure others' devices. In this article, we propose Acc, which serves as an on-demand generic discovery accelerating middleware for many deterministic neighbor discovery schemes. Acc leverages the discovery capabilities of neighbor devices, supporting both direct and indirect neighbor discoveries. Further, we present a proactive online rendezvous maintenance mechanism, which is used to reduce delays for the detection of leaving of neighbors. Our evaluations show that Acc-assisted discovery schemes reduce latency by up to 51.8\% compared to schemes consuming the same amount of energy. More importantly, to prove the real-world value of Acc, we further present and evaluate a Crowd-Alert application where Acc is employed by taxi drivers to accelerate selection of a direction with fewer competing taxis and more potential passengers, based on a 280GB dataset of more than 14,000 taxis in Shenzhen, the most crowded city in China.
\end{abstract}

Categories and Subject Descriptors: C.2.1 [Computer-Communications Networks]: Network Architecture and Design, Wireless Communication

General Terms: Design, Experimentation

Additional Key Words and Phrases: Protocol, neighbor discovery, mobile applications

ACM Reference Format:

Desheng Zhang, Tian He, Yunhuai Liu, Yu Gu, Fan Ye, Raghu K. Ganti, and Hui Lei. 2015. Generic neighbor discovery accelerations in mobile applications. ACM Trans. Sen. Netw. 11, 4, Article 63 (November 2015), 35 pages.

DOI: http://dx.doi.org/10.1145/2832914

This research was supported in part by U.S. National Science Foundation (NSF) grants CNS 0845994, CNS 1444021, CNS 1525235, CNS-1513719, NSFC 61170247, IBM OCR Fund, and the K. C. Wong Education Foundation of Hong Kong. A preliminary work was presented at ACM SenSys 2012 [Zhang et al. 2012].

Authors' addresses: D. Zhang and T. He, Department of Computer Science and Engineering, University of Minnesota, 200 Union Street SE Minneapolis, MN 55455; emails: \{zhang, tianhe\}@cs.umn.edu; Y. Liu, Third Research Institute of Ministry of Public Security, 76 Yueyang Lv, Xuhui, Shanghai, P.R.China; email: yunhuai.liu@gmail.com; Y. Gu, IBM Watson Health, 11501 Burnet Road, Austin, TX, USA 78758; email: yugu@us.ibm.com; F. Ye, Department of Electrical and Computer Engineering, Stony Brook University, 217 Light Engineering, Stony Brook, NY 11794-2350; email: fan.ye@stonybrook.edu; R. K. Ganti and H. Lei, IBM T.J. Watson Research Center, 1101 Kitchawan Road, Yorktown Heights, NY 10598; emails: \{rganti, hlei\}@us.ibm.com.

Permission to make digital or hard copies of part or all of this work for personal or classroom use is granted without fee provided that copies are not made or distributed for profit or commercial advantage and that copies show this notice on the first page or initial screen of a display along with the full citation. Copyrights for components of this work owned by others than ACM must be honored. Abstracting with credit is permitted. To copy otherwise, to republish, to post on servers, to redistribute to lists, or to use any component of this work in other works requires prior specific permission and/or a fee. Permissions may be requested from Publications Dept., ACM, Inc., 2 Penn Plaza, Suite 701, New York, NY 10121-0701 USA, fax +1 (212) 869-0481, or permissions@acm.org.

(c) 2015 ACM 1550-4859/2015/11-ART63 $\$ 15.00$

DOI: http://dx.doi.org/10.1145/2832914 


\section{INTRODUCTION}

Mobile devices, e.g., smartphones and tablets, are popular, enabling numerous applications [Ganti et al. 2011; Lane et al. 2011]. Early applications usually were built on the premise that users check into centralized servers to coordinates with peers [Google 2013; Foursquare 2013; Facebook 2013], so they typically result in excessive updating process, heavy control overhead, long communication delay, and the exposure of location information on centralized services. In contrast, new applications are proposed based on direct peer-to-peer communication [Synerge 2013; Pietiläinen et al. 2009; Softonic 2012]. Usually, they rely on data collected in an opportunistic fashion, which they process and share within a community to monitor large-scale phenomena, e.g., urban environments [Dutta et al. 2009; Dutta and Subramanian 2010], user behaviors [Yan et al. 2009; 2010], transportation [Biagnioni et al. 2011; Thiagarajan et al. 2010], and social networks [Miluzzo et al. 2011].

Many of these applications require a fast discovery of neighbor devices in a nearby region [Huang et al. 2005; Liu et al. 2004, 2010; Wikipedia 2013]. For example, fast discovery is critical for firefighters to exchange information during rescue operations [Liu et al. 2010], for players to interact with each other in location-based games [Wikipedia 2013; Nintendo 2012; Sony 2013], and for taxicabs to send status to other nearby taxicabs to enable real-time distributed dispatching [Zhang and He 2012]. This quickly collected neighbor information allows applications to effectively collaborate among participating devices.

On the other hand, in the preceding applications, radios in mobile devices are usually duty cycled between several modes to save energy or bandwidth, e.g., between an infrastructure mode and an ad hoc mode. For example, sensor nodes have to alternate their radios between an inactive mode and an active mode to save energy because most sensor nodes are powered by batteries. The duty cycling scheme prolongs the devices' lifetime; however, it poses a significant issue for the neighboring devices to find each other, as the neighboring devices may not enter the active mode at same time for a long time due to low duty cycles, e.g., $1 \%$, and thus are incapable of finding each other in time through such communication.

To address this issue, several state-of-the-art discovery protocols for wireless sensor networks [Tseng et al. 2002; Zheng et al. 2003; Dutta and Culler 2008; Kandhalu et al. 2010; Purohit et al. 2011; Bakht et al. 2012] have been proposed to achieve a bounded discovery latency. We found, however, that current protocols face two challenges when directly employed on personal devices:

-First, typical applications of sensor networks are delay tolerant, but in many mobile applications, humans are involved in the loop, and a longer latency, even though bounded, distracts user attention. One could argue that adjusting duty cycles of existing solutions [Tseng et al. 2002; Zheng et al. 2003; Dutta and Culler 2008; Kandhalu et al. 2010; Purohit et al. 2011] can reduce delay in a discovery when so desired. These schemes, however, require coordinated changes of duty cycle patterns, a requirement only suitable for the networks where a user owns the whole network and can change all devices' duty cycles collaboratively, i.e., sensor networks. In personal device networks, a user may be unable to configure key system parameters, e.g., duty cycles, of other users' devices, meaning that accelerated discovery has to be achieved only by adjusting the duty cycle of a user's own device.

-Second, many mobile applications, e.g., geosocial networking, running on personal devices desire a fast discovery only when such a need arises, unlike the sensor network applications where continuous discovery is needed to maintain network connectivity in mobile environments. Thus, we argue that allocating duty cycles continuously in advance of user demands is wasteful. 
To address the preceding two challenges, in this article we advocate accelerated discovery by individual users in an on-demand autonomous manner. In particular, we consider a scenario in which an effective discovery protocol, e.g., Disco [Dutta and Culler 2008], has already been deployed in networks running with a very low duty cycle. When a faster discovery is needed by a user, an additional energy budget (in terms of additional active slots) is used to perform an on-demand acceleration.

Our accelerator is called Acc, which functions based on knowledge collected by an existing discovery scheme. We aim at a generic middleware design that supports a wide range of discovery protocols with an arbitrary duty cycle pattern. Technically, the key novelty of $A c c$ is that it leverages knowledge in the neighbor tables of known neighbors to maximize the utility of additional on-demand energy, i.e., effectiveness of additional active slots, to accelerate discovery of unknown neighbors while also introducing no changes on any device except the discovering one. Specifically, our contributions are as follows:

-We introduce a transparent accelerating scheme Acc that works with deterministic discovery protocols to greatly accelerate the discovery process. To the best of our knowledge, this is the first work that provides an on-demand generic solution to accelerate a wide range of deterministic discovery protocols under different duty cycle patterns.

-We propose a concept of spatial-temporal coverage and define a model to quantify the effectiveness of each slot in the acceleration of discovery. The model is fully distributed and leverages only information in neighbor tables of known neighbors. It does not make any assumptions regarding radio or mobility models.

- Based on this coverage, we design an agile online scheduling algorithm to decide additional active slots under a given energy budget. Comparing our online scheduling to its theoretically optimal Oracle version, we prove that our online scheduling is competitive by obtaining its competitive ratio $\rho$, which indicates that our online scheduling algorithm has bounded performance compared to its Oracle version.

-We present a neighbor verification mechanism and a proactive rendezvous maintenance mechanism, which utilizes the online information about common neighbor reduction as a hint to infer the leaving of a neighbor, and then initiates a binary selection for additional active slots to proactively accelerate the detection process about the leaving of the neighbor.

-We test Acc at three scales of networks: (i) a small-scale testbed experiment with 11 TelosB devices, (ii) a middle-scale simulation with 100 mobile devices, and (iii) a large-scale trace driven evaluation with 14,000 vehicles. The results show that Accassisted schemes reduce the latency by up to $51.8 \%$ when consuming the same energy.

-To prove the real-world value of Acc, we propose a Crowd-Alert application to show how Acc can be employed by taxicab drivers to select a direction with fewer competing taxis or more potential passengers. We further evaluate Crowd-Alert based on a 280GB dataset consisting of 6 months of GPS traces of more than 14,000 taxis in Shenzhen, which is the most crowded city in China with 17,150 people per square kilometer [Sasin 2012]. Our application demonstrates that a smart driver increases the possibility of picking up a passenger based on an accelerated discovery, which allows drivers to quickly learn the distributions of potential passengers and competing taxicabs.

The article is organized as follows. Section 2 introduces related work. Section 3 presents background information. Section 4 provides our motivation. Section 5 proposes Acc design. Sections 6 and 7 present our implementation and simulation. Section 8 demonstrates Acc's application in a taxi dispatch system. Section 9 concludes the article. 


\section{RELATED WORK}

The neighbor discovery in low-power wireless networks has recently been studied in the literature. In general, neighbor discovery schemes can be divided into probabilistic, quorum-based, and deterministic categories:

-Probabilistic: Probabilistic protocols, e.g., the birthday protocol [McGlynn and Borbash 2001], assign different probabilities for sending, receiving, and sleeping in individual slots. Due to the birthday paradox [Mitzenmacher and Upfal 2007], such probabilistic schemes offer very good performance in the average discovery latency. But their major limitation is an unbounded worst-case discovery latency, which leads to a long tail on discovery probabilities over time. Moreover, the birthday protocol concludes that this discovery scheme aims for the stationary networks instead of the mobile networks.

-Quorum based: Quorum-based discovery protocols address the preceding unbounded latency issue by ensuring overlapping active durations between any pair of devices within a bounded time. In these schemes, time is divided into $m \times m$ continuous slots as a matrix, and each device selects one row and one column (called quorums) to become active. Therefore, regardless of which row and column a device chooses to become active, it is guaranteed to have at least two common active slots with other devices. But a main drawback of quorum-based protocols is a global parameter of $m$, which forces all devices in the network to have the same duty cycle [Tseng et al. 2002; Zheng et al. 2003]. Although some work has been proposed to support asymmetric duty cycle patterns, they can support only two different duty cycle patterns [Lai et al. 2010]. Again, quorum-based discovery protocols are also primarily proposed for stationary networks where energy is the most pressing concern, not mobility.

-Deterministic: Deterministic protocols are most closely related to our work [Dutta and Culler 2008; Kandhalu et al. 2010; Purohit et al. 2011; Bakht et al. 2012]. They recently have been proposed to handle the global parameter problem by letting every device distributedly select one or multiple prime numbers for itself to represent its duty cycle. Based on the Chinese remainder theorem [Niven and Zuckerman 1991], the devices would have bounded discovery latencies. In Disco [Dutta and Culler 2008], each device selects two prime numbers and generates its period independently based on these numbers. To improve Disco's performance, U-Connect [Kandhalu et al. 2010] proposes an activation pattern using one prime and has a shorter latency, especially in asynchronous symmetric networks. Further, WiFlock [Purohit et al. 2011] combines discovery and maintenance using a collaborative beaconing mechanism with time synchronization. Searchlight [Bakht et al. 2012] leverages the constant offset between periodic awake slots to design a simple probing-based approach to ensure discovery.

Summary. Our work presents a different design architecture than the aforementioned three categories and serves as a middleware for deterministic neighbor discovery schemes. We utilize an existing deterministic discovery protocol, e.g., Disco, to guarantee a bounded discovery latency by maintaining original active slots. Built upon the utilized protocol, our design adds only new active slots in addition to the slots specified by the utilized discovery protocol. This unique design philosophy allows an on-demand acceleration without the need for additional coordination among mobile devices. Another key novelty of this work is that when we add new active slots, we quantify the effectiveness of each added active slot on both direct and indirect discovery, and the latter part has not been considered in previous discovery designs. 


\begin{tabular}{|c|c|c|c|c|c|c|c|c|c|c|c|}
\hline $\begin{array}{l}\text { Globa } \\
\text { Time }\end{array}$ & 0 & 1 & 2 & 3 & 4 & 5 & 6 & 7 & 8 & & 10 \\
\hline$S$ & 0 & 1 & 2 & 3 & 4 & 5 & 6 & 7 & 8 & 9 & 10 \\
\hline$A$ & 0 & 1 & 2 & 3 & 4 & 5 & 6 & 7 & 8 & 9 & 10 \\
\hline 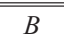 & - & - & - & 0 & 1 & 2 & $\overline{3}$ & $\overline{44}$ & $\overline{5}$ & $\overline{6}$ & 7 \\
\hline
\end{tabular}

Fig. 1. Neighbor discovery process.

\section{PRELIMINARIES FOR NEIGHBOR DISCOVERY}

In this section, we introduce some background information about how mobile devices can discover each other in a distributed network without any infrastructure support.

Note that many applications include devices with highly diverse configurations distributed in a wide geographic area, such as low-cost sensors in the wild. Therefore, it is difficult to achieve global time synchronization at fine granularity. GPS-based synchronization schemes are part of the solution [Liu et al. 2004; Jun et al. 2006], but they are typically too energy expensive to be implemented on battery-powered mobile sensors [Elson and Römer 2003] or smartphones [Paek et al. 2010]. Therefore, the devices usually decide their schedules based on a distributed yet coordinated duty cycle pattern. Specifically, to schedule its discovery, a device $S$ divides time into continuous fixed-length time slots. Then, based on a specific protocol, $S$ activates its radio and switches into a discovery mode during a specific set of slots. After that, $S$ broadcasts one or multiple discovery messages for other devices to discover its existence. At the same time, $S$ also listens to a wireless channel to receive similar messages from other devices. Essentially, when neighbor devices have overlapping slots in which they enter the discovery mode, they are able to discover each other [Dutta and Culler 2008].

Although our Acc can work with a wide range of protocols, for the sake of clarity, in this article we use Disco [Dutta and Culler 2008] as a representative example. In the evaluation, we will show how Acc works with WiFlock [Purohit et al. 2011], U-Connect [Kandhalu et al. 2010], and Searchlight [Bakht et al. 2012] as well. Specifically, Disco employs the Chinese remainder theorem [Niven and Zuckerman 1991] to guarantee a discovery latency bound. Whereas in the real implementation Disco selects two different primes for a device to solve the issue of two devices having the same prime, for simplicity we choose only one prime to represent a duty cycle of a device to show the principle of Disco. For every chosen prime number of slots, the device will enter into its discovery mode for one slot. Consequently, the actual duty cycle is equal to the reciprocal of this chosen prime number. For example, to achieve an approximately $1 \%$ duty cycle, Disco would choose the prime number of 101 . The maximal discovery latency between two devices, according to the Chinese remainder theorem, is equal to the product of two prime numbers chosen by these two devices. Figure 1 shows an example of asynchronous discoveries among three devices: $S, A$, and $B$.

In this figure, devices $S, A$, and $B$ start their local timers at global times 0,0 , and 3, respectively. According to Disco, $S$ discovers devices $A$ and $B$ at global slots 0 and 10 , respectively, based on their duty cycles, i.e., $20 \%\left(\frac{1}{5}\right), 33 \%\left(\frac{1}{3}\right)$, and $14 \%\left(\frac{1}{7}\right)$. Note that existing discovery protocols only assume that the slots at individual devices have equal lengths [Dutta and Culler 2008]. By sending two messages at the beginning and end of an active slot, they do not require aligned slots and are robust to clock drift. The perfect alignment in Figure 1 is for illustration.

The rationale behind the duty cycling-based neighbor discovery is to ensure that the distributed asynchronous devices have their active slots quickly overlapped. Without 


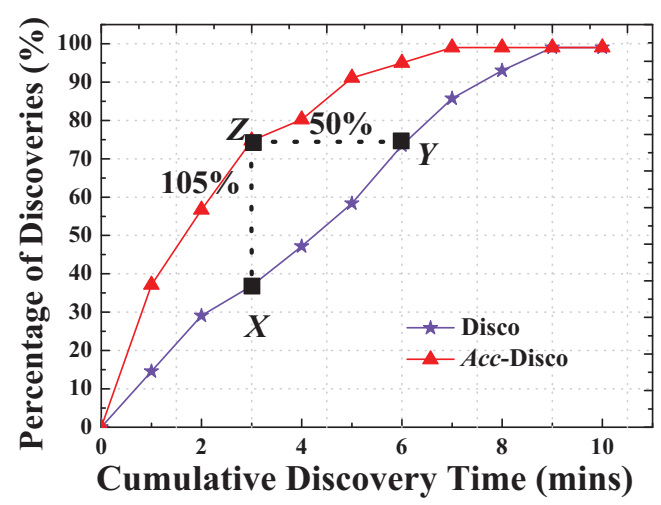

Fig. 2. Motivation.

further information, neighbor discovery protocols have to be cautious about turning nodes' radios into active slots, which may waste the energy.

\section{MOTIVATION: WHY DO WE NEED ACC?}

Our work is motivated by the observation that current state-of-the-art neighbor discovery schemes suffer from long discovery latencies due to duty cycling for energy efficiency. In many mobile applications, however, neighbor discovery has to be fast enough to enable crucial responsive user experiences. Unfortunately, for traditional discovery schemes, its design objective is to discover neighbors with a more energyefficient method, no matter how long it will take, as long as it is bounded.

We utilize a GPS dataset of 14,000 taxicabs to simulate a real-world mobile network (the detailed setting is given in Section 8) to investigate the performance of the neighbor discovery protocols. In Figure 2, we plot results on the cumulative distribution function, i.e., CDF, of latency for Disco [Dutta and Culler 2008]. As shown by point $X$, Disco discovers more than $30 \%$ of neighbors after a latency of 3 minutes; as shown by point $Y$, Disco discovers more than $70 \%$ of neighbors after a latency of 6 minutes.

Based on the preceding evaluation, we find that although such a long discovery latency ensures energy savings, it poses a significant challenge for interactive applications where energy is important but not the most pressing concern. Thus, in these applications, when needed, an on-demand fast neighbor discovery has to be performed in a very short period of time before users begin to lose their focus on the application. These observations consequently lead to a new design philosophy for neighbor discovery: to perform an on-demand fast discovery within a given additional energy budget, a device should discover its neighbors as quickly as possible to make applications function smoothly. Therefore, our design goal of Acc is to more efficiently utilize the additional energy budget to accelerate the discovery process compared to current designs with the same amount of energy.

In Figure 2, to visually show our design objective, we plot the curve of Acc-Disco, where Acc works together with Disco to accelerate the discovery. To make the comparison fair, we run Acc-Disco at the same duty cycle as Disco. But in Acc-Disco, half of the duty cycle is allocated to Disco for bounded latency and another half of the duty cycle is allocated to Acc for acceleration purposes. Therefore, Disco and Acc-Disco have the same total energy budget. The system details are given in Section 8. As shown by point $Z, A c c$-Disco discovers more than $70 \%$ of neighbors after a latency of 3 minutes. Thus, comparing point $Z$ to $X$, under the same latency, our Acc assists Disco to achieve more discoveries by a maximum of $105 \%$, whereas comparing point $Z$ to $Y$, to discover the 

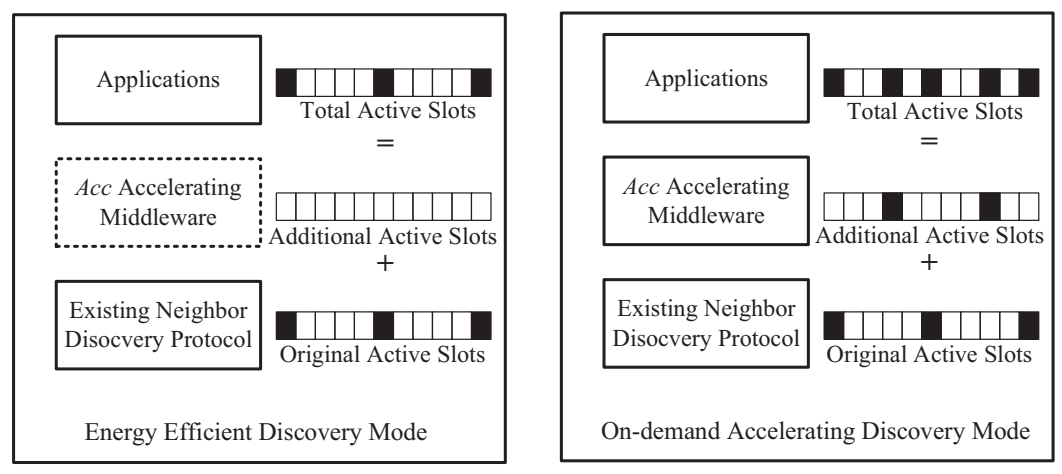

Fig. 3. Acc in the architecture.

same number of neighbors, our Acc assists Disco in accelerating its discovery process by a maximum of $50 \%$.

Based on the preceding observations, our goal is enabling Acc to optimally utilize the additional energy budget to reduce the discovery latency for the same number of neighbors rather than simply assigning this budget to the existing discovery protocols.

\section{ACC DESIGN}

In this section, we introduce our detailed design for accelerations of neighbor discovery in mobile applications.

\subsection{Main Idea}

In Figure 3, based on the location of Acc in the whole networking architecture, we introduce the main idea of $A c c$ as follows:

In this figure, an effective existing discovery protocol, e.g., Disco, has already been installed in each device. This existing protocol provides the neighbor information to the upper applications. Our Acc serves as a middleware between the existing neighbor discovery protocol and applications. Augmented further by Acc, a device runs in one of two discovery modes: energy-efficient discovery mode and on-demand accelerated discovery mode. If a fast discovery is not required, a device $S$ is in the first mode, and $A c c$ is completely transparent, i.e., a device only turns on the radio at the active slots, i.e., the black cells, indicated by the existing discovery protocol as in the left of Figure 3; otherwise, $S$ enters the second mode, concurrently performing Acc and the underlying discovery protocol for both the acceleration and the bounded latency, i.e., turning on the ratio at the active slots indicated by both the existing discovery protocol and Acc. The detailed operations of a device in these two modes are given as follows:

-Energy-efficient discovery mode: In this mode, $S$ performs the following two steps during its original active slots (as specified by the underlying discovery protocol) and turns off its radio in the rest of slots: (i) at the beginning and end of the original active slots, $S$ sends a discovery message including its neighbor table, i.e., its own duty cycle as well as IDs and duty cycles of its current known neighbors, and (ii) $S$ may receive similar discovery messages from previously unknown or known neighbors if they also become active in the same slots with $S$. Therefore, $S$ will collect some activation schedules about some known neighbors, i.e., when the known neighbors will become active again in future slots. This information is valuable because when an on-demand accelerating discovery is required, it will help $S$ decide how to accelerate the discovery. 


\begin{tabular}{|c|c|c|c|c|c|c|c|c|c|c|c|}
\hline $\begin{array}{l}\text { Global } \\
\text { Time }\end{array}$ & (b) & 1 & 2 & 3 & 4 & 5 & 6 & 7 & 8 & 9 & 108 \\
\hline$S$ & 0 & 1 & 2 & 3 & 4 & 5 & 6 & 7 & 8 & 9 & 10 \\
\hline$A$ & 0 & 1 & 2 & 3 & 4 & 5 & 6 & 7 & 8 & 9 & 10 \\
\hline$B$ & - & - & - & 0 & 1 & 2 & 3 & 4 & 5 & 6 & 7 \\
\hline
\end{tabular}

Fig. 4. Indirect discovery.

-On-demand accelerating discovery mode: When an on-demand fast discovery is required, $S$ enters this mode to accelerate the discovery with an additional energy budget. In this mode, besides original active slots, $S$ also becomes active during several additional slots to receive discovery messages. These additional slots are optimal for discovering more potential neighbors in two ways: direct neighbor discovery by $S$ itself, and indirect neighbor discovery by $S$ 's known neighboring devices. This indirect discovery is performed by receiving neighbor tables from other devices in active slots. Figure 4 gives an example of the indirect discovery.

After the discovery of a device $A$ in the global time 0 , if $S$ can select one additional active slot between the global time slot 1 to $10, S$ would select slot 6 for possible indirect discoveries via $A$, since (i) $S$ knows that $A$ will become active in slot 6 after the initial discovery, and (ii) neighbors discovered by $A$ in slot 3, e.g., $B$, will be forwarded to $S$ in slot 6 . So $S$ accelerates the discovery process of $B$ by 4 slots, i.e., from slots 10 to 6 .

A natural and key question comes up: how do we select additional active slots that are most effective when the energy budget is given? Before answering this question, we first explain the operational difference between existing discovery protocols and Acc. In existing discovery schemes, a discovering device $S$ discovers its neighbors only by $S$ itself, without any direct collaborations with neighbors already known. Therefore, when characterizing a potential active slot in terms of discovery, existing schemes may consider only how many unknown neighbors $S$ can directly discover by itself if $S$ becomes active in this slot. These direct discoveries can accelerate the discovery process on a certain level, although not significantly. In contrast, our Acc characterizes a potential active slot based on how many unknown neighbors $S$ 's known neighbors will discover can be forwarded to $S$ to achieve indirect discoveries. This indirect discovery is one of the key features of Acc. Compared to the direct discoveries, these indirect discoveries significantly accelerate the discovery process. This is because direct discoveries increase only linearly, but indirect discoveries may increase geometrically.

We break down the question of how to select additional slots into two subquestions: (i) how do we evaluate the effectiveness of all potential active slots, and (ii) among these potential active slots, how do we select a subset of active slots to maximize the discovery probability and reduce discovery latency. A potential active slot $t$ is evaluated by a metric of spatial-temporal coverage, which is considered as a slot gain to quantify discovery capabilities of all known neighbors becoming active at slot $t$. These known neighbors can discover common unknown neighbors for $S$ during the slots that $S$ is not active and then forward such information to $S$ at slot $t$. Since the known neighbors of $S$ will discover their neighbors anyway, Acc supports a transparent acceleration for $S$ running at the on-demand accelerating discovery mode. This is because no additional marginal cost, e.g., additional activations, is needed for $S$ 's neighbors running at the energy-efficient discovery mode. We present the slot gain in Section 5.2. Then we explain how to dynamically schedule a subset of active slots that maximize the total slot gains, given a fixed energy budget, i.e., the number of active slots to be added. We present this online scheduling algorithm in Section 5.3. 

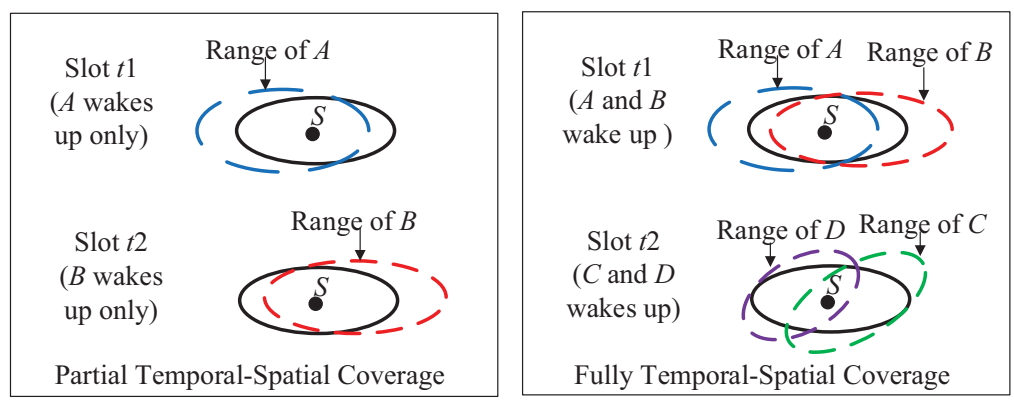

Fig. 5. Temporal-spatial coverage.

\subsection{Characterization of Slot Gain}

Before presenting the detailed characterization of the slot gain, we first provide some intuition behind this concept. To discover more unknown neighbors, a discovering device $S$ should become active at a future slot that has the largest number of potential unknown neighbors that are also becoming active. Therefore, intuitively, a future slot with more active unknown neighbors should be assigned to a larger gain.

But without making further assumptions, $S$ cannot have this information about how many unknown neighbors will become active in a certain future slot. Alternatively, $S$ indeed has information collected during the previous discoveries about how many and which kinds of $S$ 's known neighbors will become active in a certain future slot. These known neighbors will passively forward their new collected neighbor information to $S$ to achieve indirect discoveries by sending neighbor tables, if the known neighbors become active together with $S$ in a future slot. Again intuitively, a future slot with more active known neighbors should be assigned to a larger gain.

Nevertheless, we observe that not all known neighbors at $S$ are equally valuable for indirect discoveries. Specifically, $S$ should favor those important known neighbors exhibiting both temporal diversity and spatial similarity to $S$. Temporal diversity indicates that in how many slots a known neighbor is active even though $S$ is not, whereas spatial similarity indicates how likely a neighbor of a known neighbor of $S$ is also $S$ 's neighbor. Finally, a future slot with more active known neighbors exhibiting both higher temporal diversity and larger spatial similarity is assigned to a larger gain.

Note that the temporal diversity and spatial similarity of the neighbors indicate their discovering capability for the discovering devices in terms of the temporal-spatial coverage. An example of temporal-spatial coverage is given in Figure 5.

On the left side of Figure 5, we show a partial temporal-spatial coverage where the discovering device $S$ has two known neighbors $A$ and $B$, and their radio ranges are shown in the figure. Based on their waking-up schedules, $S$ is inactive in both slots $t_{1}$ and $t_{2}, A$ is active during slot $t_{1}$ only, and $B$ is active during slot $t_{2}$ only. Thus, $A$ and $B$ can only temporally and spatially cover partial neighborhood of $S$, i.e., discovering $S$ 's neighbors, when $S$ is inactive during slots $t_{1}$ and $t_{2}$. This is because during $t_{1}, A$ cannot find $S$ 's active neighbors who are inside $S$ 's range, but outside $A$ 's range; similarly during $t_{2}, B$ cannot find $S$ 's active neighbors who are inside $S$ 's range, but outside $B$ 's range. However, on the right side of Figure 5, we find a full temporal-spatial coverage for $S$ by known neighbors $A, B, C$, and $D$ in another setting. During $S$ 's inactive slots $t_{1}$ and $t_{2}$, any $S$ 's unknown active neighbor will be discovered by $A, B, C$, or $D$. The discovery result will be forwarded to $S$, when $S$ makes rendezvous with these neighbors later.

As follows, we introduce the details of how to use the temporal diversity and the spatial similarity to calculate the slot gain. 


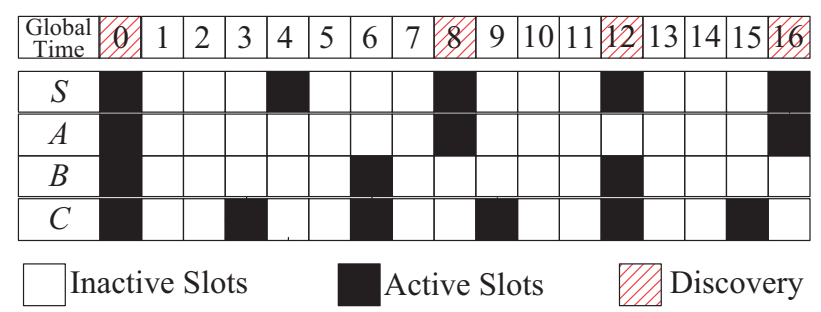

Fig. 6. Example of temporal diversity.

5.2.1. Temporal Diversity. The temporal diversity between a pair of devices $S$ and its known neighbor $A$ is determined by the difference in active slot schedules between them. The more the difference in active slots, the more likely that via $A, S$ can early indirectly discover new neighbors whom $S$ was supposed to later directly discover during $S$ 's original active slots. For example, Figure 6 shows an example of temporal diversity. In this figure, whenever $A$ becomes active, $S$ also becomes active, so the temporal diversity between them is limited. Since $A$ can only discover neighbors in the slots where $S$ also does, there is limited information that $A$ can learn but $S$ cannot. But a device $C$ frequently becomes active in the slots where $S$ is inactive, e.g., slots 3 , 6,9 , and 15 . Given a slot $t$, the more frequently $C$ becomes active before $t$, the larger the possibility that $C$ has more information on the potential neighbors not yet known to $S$. Thus, to maximize the possibility that the known neighbors can forward more information about the unknown potential neighbors to $S$, Acc attempts to activate $S$ at the slots where more known neighbors with higher temporal diversities become active.

At current slot $t_{0}$, to calculate the temporal diversity between two device $i$ and $j$ at a future slot $t$, denoted as $\alpha_{t_{0} \rightarrow t}^{(i, j)}, j$ utilizes the ratio between the number of nonoverlapping active slots between $i$ and $j$ from the current slot $t_{0}$ to slot $t$, and the total number of slots until slot $t$. This ratio is given by the following formula:

$$
\alpha_{t_{0} \rightarrow t}^{(i, j)}=\frac{\left|m_{t_{0} \rightarrow t}^{(i, i)}\right|-\left|m_{t_{0} \rightarrow t}^{(i, j)}\right|}{t-t_{0}},
$$

where $m_{t_{0} \rightarrow t}^{(i, j)}$ is the common active slot set of $i$ and $j$ from slot $t_{0}$ to slot $t$; clearly, if $j=i$, then $m_{t_{0} \rightarrow t}^{(i, i)}$ is the total active slot set of $i$ from slot $t_{0}$ to slot $t$.

In Figure 6, we show how to obtain $\alpha_{t_{0} \rightarrow t}^{(i, j)}$. Assume devices $S, A, B$, and $C$ first discover each other at slot 0 . At $t_{0}=\operatorname{slot} 1$, the temporal diversity of slot 6 for $A, B$, and $C$ to $S$ is $\alpha_{1 \rightarrow 6}^{(A, S)}=\frac{0}{5}, \alpha_{1 \rightarrow 6}^{(B, S)}=\frac{1}{5}$, and $\alpha_{1 \rightarrow 6}^{(C, S)}=\frac{2}{5}$, respectively. Clearly, $A$ has the least temporal diversity to $S$, whereas $C$ has the most temporal diversity to $S$.

5.2.2. Spatial Similarity. The spatial similarity between a pair of devices $S$ and $A$ is determined by the spatial closeness between them. In multihop networks, not all $A$ 's neighbors are $S$ 's neighbors. Intuitively, the closer $A$ is to $S$, the larger the possibility that more common neighbors exist between them. Thus, to maximize the possibility that the potential unknown neighbors forwarded by the known neighbors to $S$ are indeed $S$ 's neighbors, $A c c$ attempts to activate $S$ at slots where more known neighbors with larger spatial similarities become active.

At current slot $t_{0}$, to calculate the spatial similarity between device $i$ and $j$, denoted as $\beta_{t_{0}}^{(i, j)}, j$ utilizes the ratio between the number of common known neighbors of $i$ and itself, and the total number of known neighbors to itself at slot $t_{0}$. This ratio is given 


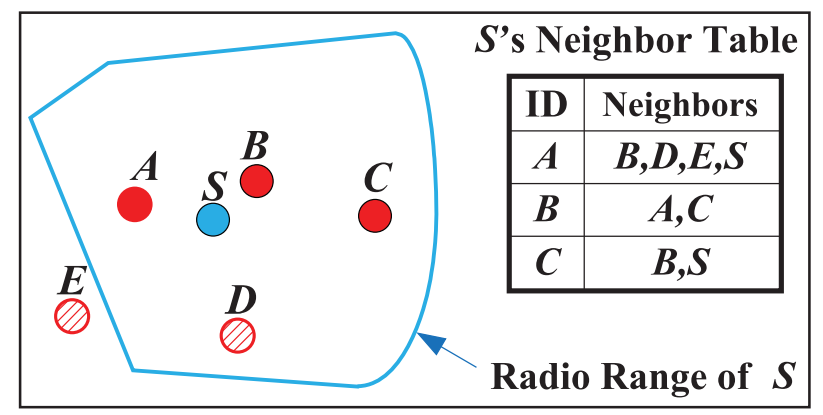

Fig. 7. Example of spatial similarity.

by the following:

$$
\beta_{t_{0}}^{(i, j)}=\frac{\left|n_{t_{0}}^{(i, j)}\right|}{\left|n_{t_{0}}^{(j, j)}\right|}
$$

where $n_{t_{0}}^{(i, j)}$ is the common known neighbor set of $i$ and $j$ at slot $t_{0}$; clearly, if $i=j, n_{t_{0}}^{(j, j)}$ is $j$ 's neighbor table at slot $t_{0}$.

Figure 7 shows an example about how to obtain $\beta_{t_{0}}^{(i, j)}$.

In this figure, at $t_{0}=$ slot 1 , among three discovered neighbors, i.e., $A, B$, and $C$, $S$ shares two, three, and two neighbors with $A, B$, and $C$, respectively, including a neighbor itself. Thus, for directly discovered neighbors $A, B$, and $C, S$ calculates $\beta_{1}^{(A, S)}=$ $\frac{2}{3}, \beta_{1}^{(B, S)}=\frac{3}{3}$ and $\beta_{1}^{(C, S)}=\frac{2}{3}$. For indirectly discovered neighbors, e.g., $D, S$ calculates $\beta_{1}^{(D, S)}=\frac{1}{3}$, since only one, i.e., device $A$, out of three known neighbors of $S$ has $D$ in its neighbor table.

5.2.3. Slot Gain Calculation. Based on the preceding observations, a discovering device $S$ assigns larger gains to the slots that have more active devices with higher temporal diversity and larger spatial similarity. At current slot $t_{0}$, based on Equations (1) and (2), $S$ calculates the slot gain of slot $t$, denoted as $\gamma_{t_{0} \rightarrow t}^{(S)}$, as follows:

$$
\gamma_{t_{0} \rightarrow t}^{(S)}=\sum_{i \in n_{t_{0}}^{(S, S)}} \alpha_{t_{0} \rightarrow t}^{(i, S)} \beta_{t_{0}}^{(i, S)}=\sum_{i \in n_{t_{0}}^{(S, S)}} \frac{\left(\left|m_{t_{0} \rightarrow t}^{(i, i)}\right|-\left|m_{t_{0} \rightarrow t}^{(i, S)}\right|\right) \times\left|n_{t_{0}}^{(i, S)}\right|}{\left(t-t_{0}\right) \times\left|n_{t_{0}}^{(S, S)}\right|}
$$

where $n_{t_{0}}^{(S, S)}$ is the neighbor table of $S$ at slot $t_{0}$.

Ideally, if $S$ is required to discover all of its neighbors becoming active from slot $t_{0}$ to $t$ but without being active all the time, then $S$ should select a set of known neighbors who can cover the entire radio range, i.e., spatial coverage, of $S$ from slot $t_{0}$ to $t$, i.e., temporal coverage, such as the fully temporal-spatial coverage on the right side of Figure 5. The temporal coverage is easy since we select a neighbor subset, if any, that has neighbors continuously becoming active from slot $t_{0}$ to $t$. But without further assumptions regarding a device's radio model, the spatial coverage is hard to perform. Essentially, $S$ could use its complete neighbor set to represent its radio area, but $S$ does not know its complete neighbor set either-only a partial known neighbor set at a specific slot. Therefore, we employ $S$ 's partial known neighbor set, i.e., $n_{t_{0}}^{(S, S)}$, to represent its radio area, i.e., the spatial coverage for $S$ is the coverage of $S$ 's known neighbor set. This strategy performs best in the situation where the partial known neighbor set is uniformly distributed in the complete neighbor set. 
Consequently, the denominator $\left(t-t_{0}\right) \times\left|n_{t_{0}}^{(S, S)}\right|$ in the last term of Equation (3) is the temporal-spatial coverage that should be provided for $S$ to discover all of its neighbors becoming active from slot $t_{0}$ to $t$, whereas the numerator $\left(\left|m_{t_{0} \rightarrow t}^{(i, i)}\right|-\left|m_{t_{0} \rightarrow t}^{(i, S)}\right|\right) \times\left|n_{t_{0}}^{(i, S)}\right|$ is the temporal-spatial coverage that a known neighbor $i$ can provide for $S$. Therefore, the fraction represents, among the total temporal-spatial coverage of $S$, how much coverage can be provided by $i$ who becomes active in slot $t$. This is the physical meaning of slot gains.

For example, with the schedule in Figure 6 and the neighbor table in Figure 7, assuming that $t_{0}$ is slot 1 , a discovering device $S$ calculates slot 6 's slot gain according to the follow formula:

$$
\gamma_{1 \rightarrow 6}^{(S)}=\alpha_{1 \rightarrow 6}^{(A, S)} \beta_{1}^{(A, S)}+\alpha_{1 \rightarrow 6}^{(B, S)} \beta_{1}^{(B, S)}+\alpha_{1 \rightarrow 6}^{(C, S)} \beta_{1}^{(C, S)}=\frac{0}{5} \frac{2}{3}+\frac{1}{5} \frac{3}{3}+\frac{2}{5} \frac{2}{3}=\frac{7}{15} .
$$

\subsection{Online Activation Scheduling}

In the previous section, we presented the method to calculate the slot gains for all the slots based on the neighbor table of a discovering device. According to the obtained slot gains, in this section we first present our online scheduling algorithm, given a fixed duty cycle budget $\mathbb{B}$. This online algorithm outputs a slot sequence for additional activations and updates this sequence consistently based on the latest yet incomplete neighbor table. Then by comparing this online algorithm to its optimal Oracle version, we theoretically analyze the proposed algorithm to show its performance via a concept called competitive ratio.

5.3.1. Scheduling Algorithm. In our scheduling algorithm, a discovering device $S$ decides an additional active slot sequence $\mathbb{A} \mathbb{S}$, which includes several additional active slots, according to three inputs as follows:

(i) Additional energy budget $\mathbb{B}$. Given $\mathbb{B}$ in terms of additional duty cycles, e.g., $\frac{2}{11}$ beyond what has already been consumed by an underlying discovery scheme, $S$ performs discoveries in some additional slots. $\mathbb{B}=\frac{2}{11}$ indicates that on average every 11 slots, $S$ can additionally become active in 2 slots besides the original active slots.

(ii) Neighbor table $n_{t_{0}}^{(S, S)}$ in current slot $t_{0}$. After every active slot, $n_{t_{0}}^{(S, S)}$ will be updated based on latest neighbor information collected during this active slot. With this updated $n_{t_{0}}^{(S, S)}, S$ continues to decide upon following additional active slots based on the updated slot gains we defined in Equation (3).

(iii) Next original active slot $t_{N}$. We take $t_{N}$ into consideration because $S$ should not select additional active slots after $t_{N}$. This is because all slot gains may be changed after $t_{N}$, since $S$ 's neighbor table may be changed after an active slot. Therefore, selecting additional active slots after $t_{N}$ will lead to a suboptimal selection.

The preceding three inputs provide necessary information for $S$ to decide $\mathbb{A} S$ with Algorithm 1 after every active slot.

Figure 8 gives an example of this algorithm.

Suppose that $t_{0}=1$, the original duty cycle is $\frac{1}{11}$, and $\mathbb{B}$ is $\frac{2}{11}$, which means that in every 11 slots, $S$ can activate approximately two additional active slots. Suppose that slots 3 and 10 have the top two largest gains among all slots before $t_{N}=$ slot 11 . Therefore, in the first round, $S$ selects slots 3 and 10, and puts them into $\mathbb{A} S$. After the activation in slot $3, S$ updates the slot gains of remaining slots via $n_{3}^{(S, S)}$. Suppose that now slot 6 has the largest slot gain, instead of slot 10, so in the second round, $S$ would select slot 6 as the last additional slot to update $\mathbb{A} \mathbb{S}$. 


\begin{tabular}{|c|c|c|c|c|c|c|c|c|c|c|c|c|}
\hline \multirow{2}{*}{$\begin{array}{c}\text { Selection of } 2 \\
\text { extra active } \\
\text { slot in Slot } 1\end{array}$} & 1st Cycle & 0 & |1 || & 2 & 3 & 4 & 5 & 6 & 7 & 8 & 9 & 0 \\
\hline & 2nd Cycle & 11 & 12 & 13 & 14 & 15 & 16 & 17 & 18 & 19 & 20 & 21 \\
\hline \multirow{2}{*}{$\begin{array}{l}\text { Selection of } 1 \\
\text { extra active } \\
\text { slot in Slot } 3\end{array}$} & 1st Cycle & 0 & 1 & 2 & B & 4 & 5 & 6 & 7 & 8 & 9 & 10 \\
\hline & 2nd Cycle & 11 & 12 & 13 & 14 & 15 & 16 & 17 & 18 & 19 & 20 & 21 \\
\hline $\begin{array}{c}\text { Current } \\
\text { Slot }\end{array}$ & & & & & & & & & & & $\begin{array}{l}\text { Idi } \\
\text { tive }\end{array}$ & $\begin{array}{l}\text { onal } \\
\text { Slots }\end{array}$ \\
\hline
\end{tabular}

Fig. 8. Example of activation scheduling.

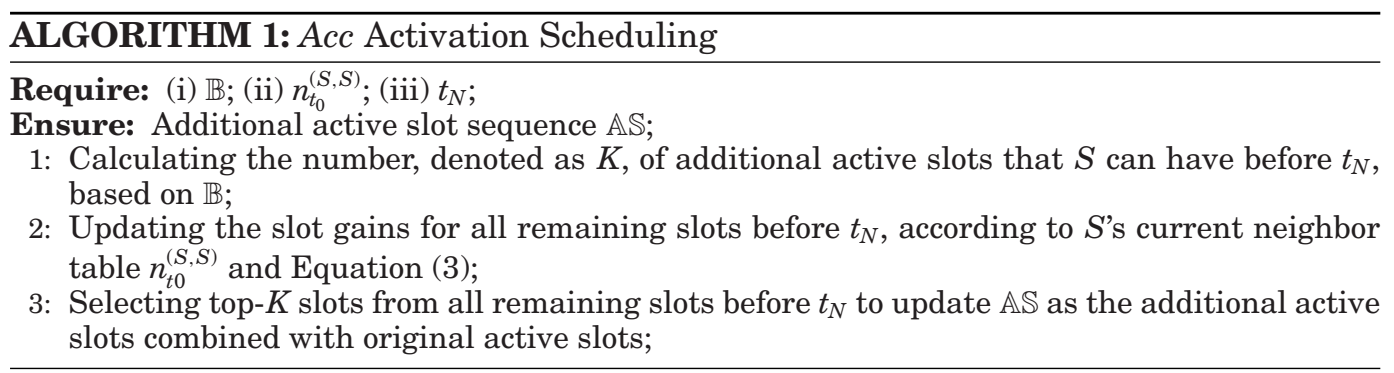

5.3.2. Competitive Analysis of Scheduling Algorithm. We analyze the performance of our online scheduling algorithm by comparing it to its optimal Oracle version. In our online scheduling, $S$ 's incomplete neighbor table in slot $t_{0}, n_{t_{0}}^{(S, S)}$, is processed piece by piece in a serial fashion to decide $\mathbb{A} \mathbb{S}$, because it is consistently updated, whereas the Oracle version will have the complete neighbor table $N^{(S, S)}$, not $n_{t_{0}}^{(S, S)}$, to decide AS. In the appendix, we prove that our online scheduling is competitive by showing that the performance ratio between it and its Oracle version, denoted as $\rho$, is bounded by a parameter $R$, which is the size ratio between $n_{t_{0}}^{(S, S)}$ and $N^{(S, S)}$. The rationale behind this analysis is that our online scheduling performance is proportional to the size of $n_{t_{0}}^{(S, S)}$. For example, if $R=1$, then our online algorithm is as effective as its Oracle version, as $R=1$ indicates that $n_{t_{0}}^{(S, S)}=N^{(S, S)}$.

\subsection{Neighbor Verification}

In the previous section, we introduce how to use online activation scheduling to accelerate the process of neighbor discovery by indirect discovery. In the scenario of mobile multihop networks, for a discovering device, a neighbor's neighbor may not be its neighbor when the discovering device indirectly discovers it. This discovery would be a false positive. Therefore, we propose a passive neighbor verification technique to verify whether indirectly discovered neighbors are actually one-hop neighbors.

In this article, we define a neighbor of a device $S$ as a device who is continuously in the communication range of $S$ at least a time period $p$, which is the discovery latency bound of an underlying neighbor discovery scheme. Two devices just transitorily were in communication ranges of each other cannot be seen as neighbors, as they cannot be discovered by each other. Therefore, a neighbor will be discovered by Acc in advance or by an underlying protocol eventually. If two devices discover each other and then move out of communication ranges of each other within a time period $p$, then they are not considered to be neighbors (false position) and will be removed.

During a discovery process, since every device would broadcast its neighbor table to its neighbors during the discover process, a discovering device would have duty cycle 


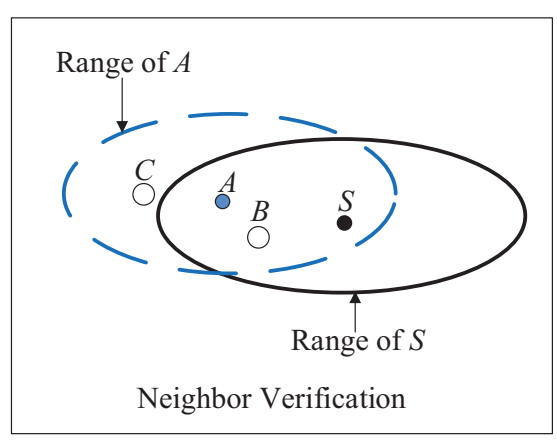

Fig. 9. Neighbor verification.

patterns of indirectly discovered neighbors whether they are one-hop neighbors or twohop neighbors. Based on these duty cycle patterns, the discovering device would know when an indirectly discovered neighbor will become active and broadcast messages to its neighbors. Therefore, in our passive neighbor verification, the discovering device would become active in the active slots of every indirectly discovered neighbor and listen to the channel for its messages for a time period of $p$. If the discovering device receives messages from this neighbor for a time period of $p$, then it indicates that this indirectly discovered neighbor is an actual one-hop neighbor. In contrast, if the discovering device does not receive messages from this neighbors for a time period of $p$, then it indicates that this indirectly discovered neighbor is not an actual one-hop neighbor.

Figure 9 gives an example of the neighbor verification process for indirectly discovered neighbors. Assume that we have a discovering device $S$. Based on its direct neighbor $A$, the discovering device $S$ indirectly discovers two neighbors, i.e., $B$ and $C$. $B$ is a one-hop neighbor of $S$, and $C$ is a two-hop neighbor of $S$. Based on the duty cycle patterns of $B$ and $C, S$ also becomes active during active slots of $B$ and $C$, after the initial indirect discovery of them. During these active slots of $B$ and $C, S$ tries to receive their messages passively, in addition to its own active slots. Because $B$ is within $S$ 's communication range and $C$ is out of $S$ 's communication range, $S$ can receive the message from $B$, but not from $C$. Therefore, $S$ has verified that $B$ is a one-hop neighbor and that $C$ is a two-hop neighbor.

\subsection{Proactive Online Rendezvous Maintenance}

Neighbor discovery is a process for identifying neighboring devices so that a device $S$ can send messages to other devices in its neighborhood, whereas rendezvous maintenance is a process in which $S$ makes contact with its discovered neighbors regularly to verify and maintain the neighboring relationship by timely detecting if the neighbors are still in the neighborhood. But such a discovered neighborhood relationship among $S$ and its neighbors is only temporal in mobile applications, because both the neighbors and $S$ are moving around and will leave the radio ranges of each other after a period of time. The leaving of a known neighbor $A$ is detected by $S$ through a failure to receive the discovering message from $A$ in the slot where $S$ and $A$ both become active, according to the schedule obtained when they first discover each other. After such a failure, $S$ just drops off $A$ from its neighbor table. The preceding scheme is the normal rendezvous for a device $S$ to keep its neighbor table up-to-date in existing protocols where the rendezvous is treated as a "rediscovery" during which a device and its known neighbors are both in the active slots again. 

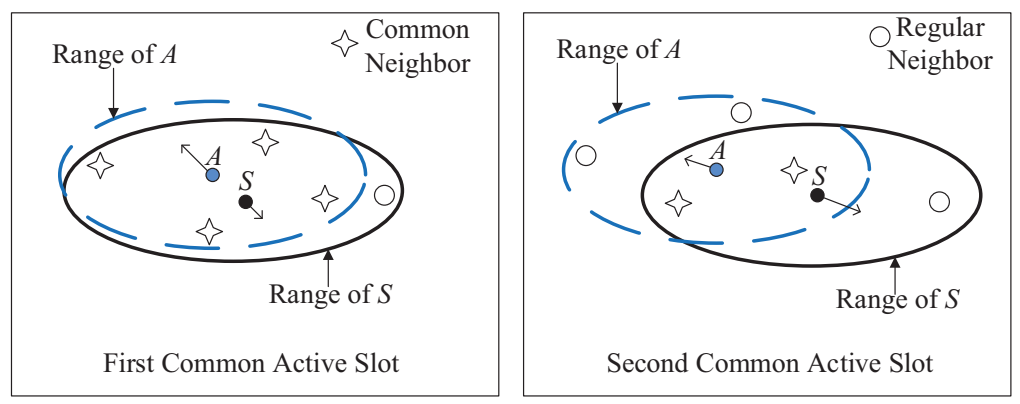

Fig. 10. Reduction of common neighbors.

In this work, we argue that this passive rediscovery-based rendezvous takes a long time delay to detect the fact that a neighbor $A$ has already left $S$ 's ratio range, e.g., a detecting delay for two devices with $1 \%$ duty may take up to $101 \times 101$ slots. Such a long delay may be acceptable for delay-tolerant applications, e.g., sensor networks, but it is typically not acceptable in the interactive applications where the leaving of a neighbor should be proactively detected as soon as possible, instead of passively depending on the rediscovery. In our $A c c$, we proactively maintain the rendezvous to reduce the detecting delay in an on-demand method if it is required by users.

The rationale of our online rendezvous maintenance mechanism is as follows. We divide the rendezvous maintenance into two subobjectives: when to proactively maintain the rendezvous and how to proactively maintain the rendezvous. Since our Acc is designed as a transparent middleware, we do not change the schedule of neighboring devices. Thus, a naive yet safe method is that right after the discovering device $S$ discovers a neighbor $A, S$ becomes active in every slot where $A$ becomes active to verify whether $A$ is still a neighbor of $S$. This naive method is the quickest method to transparently detect the leaving of $A$ without the cooperation from $A$, but this method involves too much energy consumption for $S$, as $S$ has to wake up at every active slot of $A$.

To address this issue, in Acc, $S$ first uses the reduction of the common neighbors of $S$ and $A$ between two normal rendezvous as a hint to initiate a proactive rendezvous maintenance regarding $A$. After the beginning of the maintenance, $S$ utilizes a binary selection to find the some active slots of $A$ for the additional wake-ups to quickly detect whether $A$ leaves the range of $S$. If $A$ is still in $S$ 's range but the reduction of the common neighbors continues, $S$ continues to select additional wake-up slots until $A$ leaves the range of $S$ or the reduction stops. In the following, we give details about when and how to process the proactive rendezvous maintenance.

5.5.1. When to Initiate Proactive Online Rendezvous Maintenance. After the initial discovery of $A$, if the proactive online rendezvous maintenance is required by users, $S$ compares the common neighbors between itself and $A$ after every normal rediscovery about $A$. If $S$ detects a reduction of the common neighbors, $S$ initiates the proactive rendezvous maintenance regarding $A$. Figure 10 gives an example of the reduction of the common neighbors for a discovering device $S$ and its neighbor $A$.

In this figure, in the first common active slot, $S$ and $A$ have four common neighbors, whereas in the second common active slot, $S$ and $A$ have only two common neighbors (due to the movements of $S$ and $A$ ). The reduction can be obtained by their neighbor tables that they broadcasted in the common active slot. Such a reduction of the common neighbors indicates that $A$ is leaving the range of $S$. Thus, $S$ initiates the proactive rendezvous maintenance regarding $A$ after the second common active slot. The 


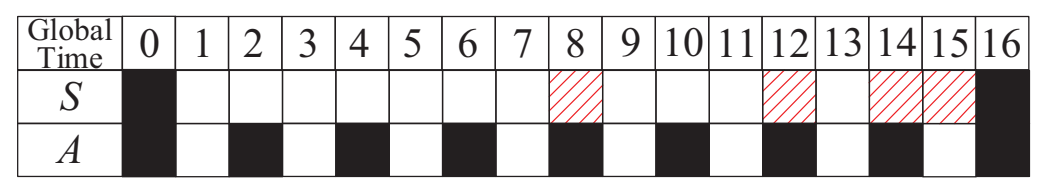

\section{Inactive Slots Active Slots Madditional Wakeup}

Fig. 11. Selection of additional wake-up for rendezvous maintenance.

rationale between method is that the fewer the common neighbors, the farther the distance between $S$ and $A$, and the more likely $A$ is leaving the range of $S$.

5.5.2. How to Initiate Proactive Online Rendezvous Maintenance. In the rendezvous maintenance regarding $A, S$ selects some active slots of $A$ for the additional wake-up (before the next normal common active slot) to reduce the detecting delay for the fact that $A$ leaves the radio range of $S$. We utilize a binary selection to choose these slots. Figure 11 gives an example of $S$ selecting additional wake-up slots to detect the leaving of $A$.

In this figure, $S$ detects the reduction of common neighbors between $S$ and $A$ at slot 0 , and initiates the proactive online rendezvous maintenance. Further, we assume a situation where the reduction of common neighbors between $S$ and $A$ is continuously detected:

$-S$ calculates $A$ 's active slots, i.e., slots $2,4,6,8,10,12$, and 14 , before the next normal rediscovery at slot 16 ;

$-S$ utilizes a binary selection to obtain slot 8 as an additional wake-up, which is in the middle of current slot 0 and the next normal rediscovery at slot 16 ;

-After waking up at slot $8, S$ continues to detect the reduction of the common neighbors, so $S$ utilizes the same binary selection again to obtain slot 12 as an additional wake-up, which is in the middle of current slot 8 and the rediscovery slot 16;

-After waking up at slot $12, S$ selects slot 14 as an additional wake-up, which is in the middle of current slot 12 and the normal rediscovery slot 16 ;

- Similarly, after waking up at slot $14, S$ selects slot 15 as an additional wake-up, which is in the middle of current slot 14 and the normal rediscovery slot 16;

-This process continues until no reduction of common neighbors is detected or after $A$ leaves the radio range of $S$; and

-Finally, $S$ drops off $A$ from its neighbor table if $A$ leaves the radio range of $S$.

In the preceding method, $S$ is based on the online information about the reduction of the common neighbors to proactively accelerate the rendezvous maintenance. The rationale behind this method is that the fewer the common neighbors, the more likely $A$ leaves the radio range of $S$.

Note that even without the preceding proactive online rendezvous maintenance, our regular accelerated discovering process by Acc implicitly expedites the delay of the detection for the fact that a neighbor leaves the radio range of a discovering device. This is because a discovering device will wake up more in the active slots of the known neighbors for the indirect discovery, according to the design of Acc. Thus, if a known neighbor of $S$ is not broadcasting in the active slot when it is supposed to be, then $S$ removes this neighbor from the neighbor table, which enables $S$ to more quickly detect the leaving of its neighbors, although the introduced proactive online rendezvous maintenance can further accelerate the detection. 


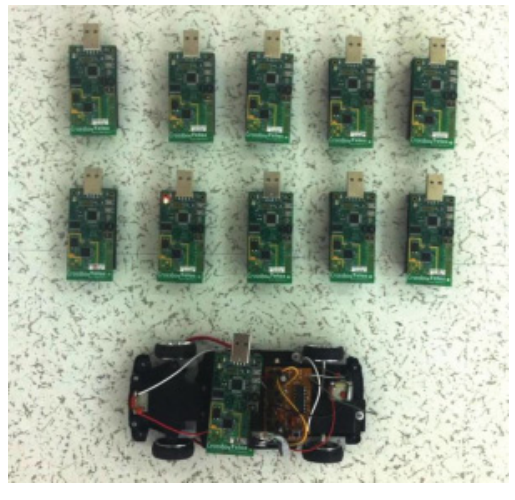

Fig. 12. Testbed setup.

\begin{tabular}{|c|c|c|}
\hline Energy & $\begin{array}{c}\text { Additional } \\
\text { Duty Cycle }\end{array}$ & $\begin{array}{c}\text { Original } \\
\text { Duty Cycle }\end{array}$ \\
\hline Disco & $\begin{array}{c}\text { Used by } \\
\text { Disco }\end{array}$ & $\begin{array}{c}\text { Used by } \\
\text { Disco }\end{array}$ \\
\hline Base-Disco & $\begin{array}{c}\text { Used by } \\
\text { Baseline }\end{array}$ & $\begin{array}{c}\text { Used by } \\
\text { Disco }\end{array}$ \\
\hline Acc-Disco & $\begin{array}{c}\text { Used by } \\
\text { Acc }\end{array}$ & $\begin{array}{c}\text { Used by } \\
\text { Disco }\end{array}$ \\
\hline
\end{tabular}

Fig. 13. Compared schemes.

\section{TESTBED EVALUATION}

To evaluate $A c c$ in a real-world setting, we integrate Acc with two state-of-the-art discovery protocols: Disco [Dutta and Culler 2008] and WiFlock [Purohit et al. 2011]. To verify whether the accelerated neighbor discovery would perform well on resourceconstrained sensor nodes, we implement the preceding two schemes employing 11 TelosB sensor devices with a 10KB RAM size on the TinyOS/Mote platform. During the testbed experiments, we deploy 10 TelosB sensor devices in a one-hop grid network and utilize a mobile toy car attached with another TelosB as a discovering device, with a mobility pattern of circling around the grid. This mobile node introduces the relative mobility between a discovering device and its neighbors, which is to verify that the mobility would not affect the neighbor discovery itself. The testbed is shown in Figure 12.

For individual devices, we set the time slot length at $25 \mathrm{~ms}$ for two reasons: (i) for direct discovery, a smaller slot leads to a faster discovery, but a too-small slot $(<5 \mathrm{~ms})$ leads to the jitters introduced by the TinyOS timer library [Dutta and Culler 2008], and (ii) for indirect discovery, a bigger slot reduces collisions of messages and enables more exchanges of neighbor tables. Based on the preceding two reasons, we make a trade-off about time slot length at $25 \mathrm{~ms}$. Note that WiFlock was implemented on modified hardware to support an extremely small time slot of $80 \mu \mathrm{s}$ [Purohit et al. 2011], but in our work we implement WiFlock only on a standard hardware to examine the principle of its collaborative beaconing mechanism. In our experiment, all schemes have the same energy budget (both original and additional) for devices to ensure a fair comparison. But different schemes use the same energy budget differently in terms of selecting active slots. The additional duty cycle budget $\mathbb{B}$ for the acceleration is set to be $5 \%$, the same as the original duty cycle of $5 \%$ on every device. The $5 \%$ duty cycle is extensively studied in Disco [Dutta and Culler 2008].

To evaluate the effectiveness of the slot gains we proposed, we also implemented a baseline design. This design shares the same scheduling scheme as Acc, but it uses the number of active devices in a slot $t$ as the slot gain, not considering any temporal diversity or spatial similarity. Thus, we implement three versions as shown in Figure 13. In all three versions, the original duty cycle is controlled by Disco, and the additional duty cycle is controlled by its own schemes. Similar versions are implemented for WiFlock.

We evaluate the preceding schemes by three metrics: (i) the percentage of discoveries with respect to cumulative discovery time, (ii) the number of discovered devices in different time intervals, and (iii) the average discovery latency in different duty cycles. The first two metrics are to verify the effect of Acc's assistance to the existing schemes 


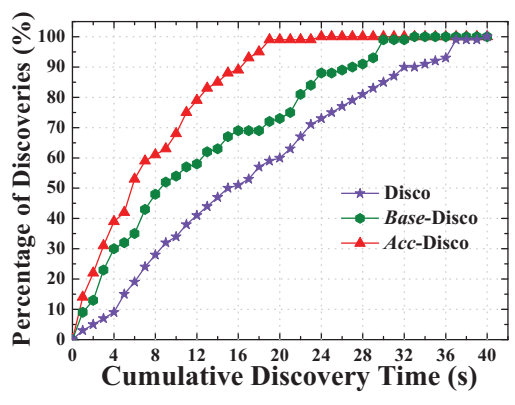

Fig. 14. Disco CDF.

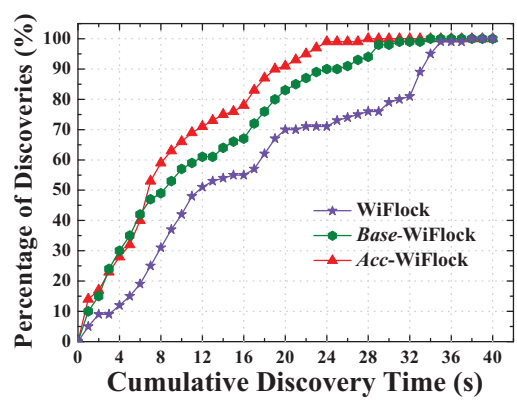

Fig. 15. WiFlock CDF.

in the acceleration of discovery process in Section 6.1. The third metric is to verify the effect of different duty cycles on the average discovery latency in Section 6.2.

In an experiment, after every 40 slots, i.e., about 1 second, the discovering device logs the number of neighbors it discovered so far. All experiments are repeated 20 times, and the average results are reported.

\subsection{Effectiveness in Acceleration of Discovery}

Figure 14 plots the acceleration effect of Disco. In this figure, we observe that the curve of Acc-Disco is above all other curves in every percentage of discoveries. For example, to discover $80 \%$ of neighbor devices, Acc-Disco, Base-Disco, and Disco spend around 13, 22, and 27 seconds, respectively. Acc-Disco finishes the discovery process faster than Disco by $51.8 \%$, whereas both consume the same energy. This is because Disco does not consider using known neighbors to discover unknown neighbors, which leads to a longer discovery process in which a device has to find its neighbors one by one. In addition, we observe that Base-Disco outperforms the original scheme by a maximum of $18.9 \%$ when discovering more than $99 \%$ of neighbors on average. This is because Base-Disco selects active slots with more known neighbors becoming active, which proves the value of taking the known neighbors into consideration. But we also observe that Acc-Disco still outperforms Base-Disco by nearly $36.6 \%$ when discovering more than $99 \%$ of neighbors. This suggests that when selecting additional active slots, considering only the quantity, not the quality, of devices becoming active in slots is not enough to significantly accelerate discovery. This can also be shown by the fact that Base-Disco discovers half of devices' neighbors by 8 seconds but finishes the whole discovery process at 32 seconds. The preceding results indicate that Acc-Disco exhibits a significant acceleration when compared to other versions.

In Figure 15, we observe similar results as in Figure 14. Among the three versions, $A c c$-WiFlock achieves the highest performance in the percentage of discovered devices in most instances of the discovery process. But we also observe that the performance gain between $A c c$-WiFlock and other versions of WiFlock is less than that between Acc-Disco and other versions of Disco. This is because in the collaborative beaconing mechanism of WiFlock, WiFlock has already taken neighbor tables into consideration. Different from Acc-WiFlock and Base-WiFlock, however, the neighbor tables in WiFlock are intended to maintain the membership of a device group to achieve synchronized listening. In Figure 15, we observe that Base-WiFlock outperforms WiFlock as well. This demonstrates the effectiveness of considering known neighbors for unknown neighbor discovery. But the fact that Acc-WiFlock outperforms Base-WiFlock indicates that considering temporal-spatial coverage, instead of only the number of neighbors, achieves further improvement. This is because by simply measuring the slot gain as the number 


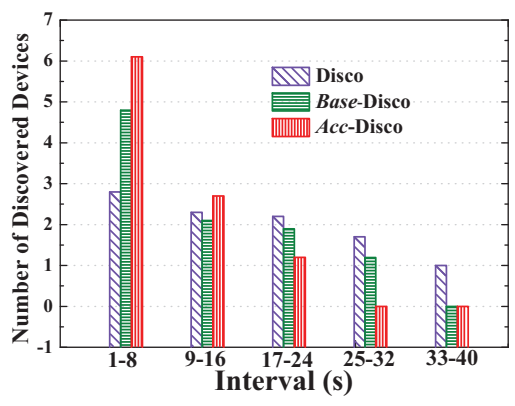

Fig. 16. Disco distribution.

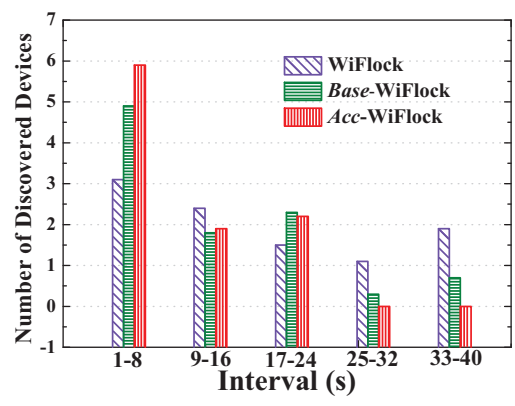

Fig. 17. WiFlock distribution.

of active neighbors, Base-WiFlock increases performance to a certain level but cannot make a device become active at the most effective slots, as Acc-WiFlock does.

Figures 16 and 17 plot the number of neighbors discovered in every 8-second time window under the versions of Disco and WiFlock. These two figures provide the distribution of discovered neighbor numbers in different phases of the discovery process. From Figures 16 and 17, we observe that both Acc-Disco and Acc-WiFlock discover the largest number of neighbor devices during the first 8 seconds. In contrast, the other versions discover relatively uniform numbers of devices over time. The reason for Disco's uniform discoveries is obvious, as Disco performs a pair-wise discovery where discovering more neighbors is not helpful for the discovery of the next neighbor. But WiFlock indeed considers a group-based strategy. One explanation for WiFlock's uniform discoveries is that WiFlock's synchronized listening and one-way discovery mechanism are efficient only for an existing group of devices to discover a new device, not for a new device to discover all of its neighbors.

From the preceding four figures, we conclude that when an additional energy budget is given for an acceleration of the discovery process, considering the number of devices active in a slot (baseline) can assist current discovery schemes to a certain level, but there still is room to improve. By taking different qualities of known neighbors into consideration, i.e., the temporal diversity or spatial similarity of neighbors, Acc further accelerates the discovery process.

\subsection{Impact of Duty Cycle}

Figures 18 and 19 plot the impact of two different original duty cycles on average discovery latencies in both Disco and WiFlock. The average discovery latency is defined as the time a device takes to discover all of its neighbors divided by the number of its neighbors. We observe that the versions with Acc outperform the versions with Baseline and original schemes by a maximum of $42.1 \%$ and $53.8 \%$, respectively. We also observe that the performance gain between the Acc-assisted versions and original versions increases as the duty cycle increases. In Disco, this gain increases from $47.7 \%$ to $53.8 \%$, whereas in WiFlock, it increases from $39 \%$ to $47.3 \%$. This indicates that as devices become active more frequently, a discovering device can obtain more information from its known neighbors by considering the temporal diversity or spatial similarity of neighbors. Again, the performance gain between the Acc-assisted version and the original version in WiFlock is smaller than that in Disco, which is also because of WiFlock's collaboration beaconing scheme. We observe different trends in the performance gain between Acc- and baseline-assisted versions in different protocols. The gain between Acc-Disco and Base-Disco decreases from $42.1 \%$ to $37.9 \%$, whereas that between Acc-WiFlock and Base-WiFlock increases from 29.4\% to $33.3 \%$. 


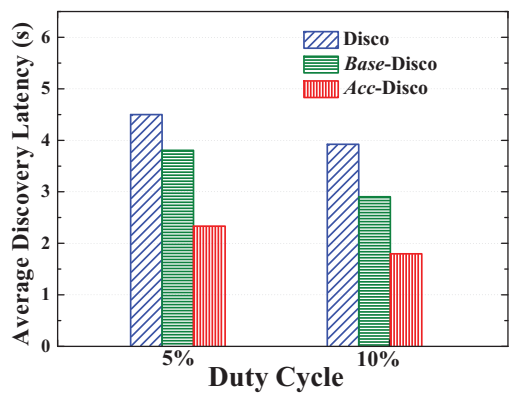

Fig. 18. Disco latency.

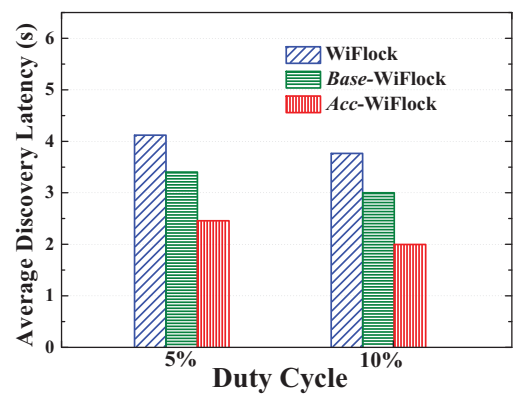

Fig. 19. WiFlock latency.

This indicates that the slot gains utilized by baseline and Acc have different effects in different protocols.

It also shows that the performance gain between $A c c$ - and Base-WiFlock, i.e., $29.4 \%$, is smaller than the gain in Disco-related comparisons, i.e., 42.1\%. This result is consistent with the observation that the performance gain between the Acc-assisted and the original version in WiFlock, i.e., 39\%, is smaller than that in Disco, i.e., $47.7 \%$. Note that even with double duty cycles, the average discovery latency does not reduce significantly in all three protocols. This is because by increasing duty cycles, Disco guarantees the proportionally reduced worst-case latency instead of the average latency.

From Figures 18 and 19, we conclude that when devices become more active, Acc more effectively assists the discovering device in accelerating the discovery process by leveraging the known neighbors to discover unknown neighbors.

\section{SIMULATION EVALUATION}

To evaluate Acc serving as an accelerating middleware to support different protocols in larger-scale networks, we simulate Acc with four discovery protocols: Disco [Dutta and Culler 2008], U-Connect [Kandhalu et al. 2010], WiFlock [Purohit et al. 2011], and Searchlight [Bakht et al. 2012]. In our 30-minute simulation, 100 mobile devices are uniformly deployed in a square area of $200 \mathrm{~m} \times 200 \mathrm{~m}$. The radio ranges of devices are set from $20 \mathrm{~m}$ to $100 \mathrm{~m}$, which lead to average device densities from 3.6 to 55.36. We use a nontrivial pure random waypoint model as a mobility model [Alparslan and Sohraby $2007]$, with an average velocity of $1 \mathrm{~m} / \mathrm{s}$. In addition to the metrics that we investigate in the testbed experiment, we evaluate the rendezvous maintenance in Section 7.4.

Note that in a mobile multihop network, neighboring relations are consistently changing, and it is extremely costly in terms of energy to keep neighbor tables upto-date, i.e., immediately discovering a device when it is in one device's communication range. In the evaluation, we define a neighbor of a device $A$ as a device continuously in the communication range of $A$ at least for a time period $p$, which is the discovery latency bound of an underlying neighbor discovery scheme. Two devices just transitorily in communication ranges of each other cannot be seen as neighbors, as they cannot be discovered by each other. Therefore, a neighbor will be discovered by Acc in advance or by an underlying protocol eventually. If two devices discover each other and then move out of communication ranges of each other within a time period $p$, then they are not considered as neighbors (false position) and will be removed. In our experiment, all schemes have the same energy budget (both original and additional) for devices to ensure a fair comparison. But different schemes use the same energy budget differently in terms of selecting active slots. We test Acc with two metrics, i.e., the percentage of discoveries and discovery latency. For both of them, the discovery delay is calculated 


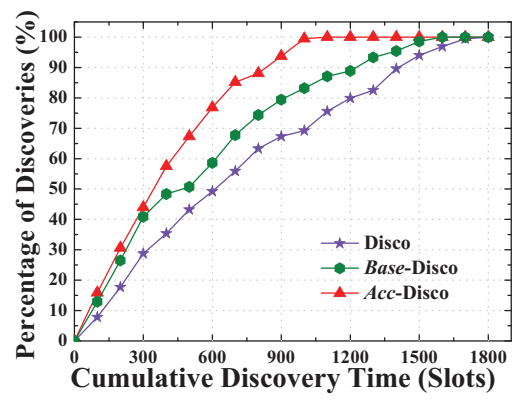

Fig. 20. Disco CDF.

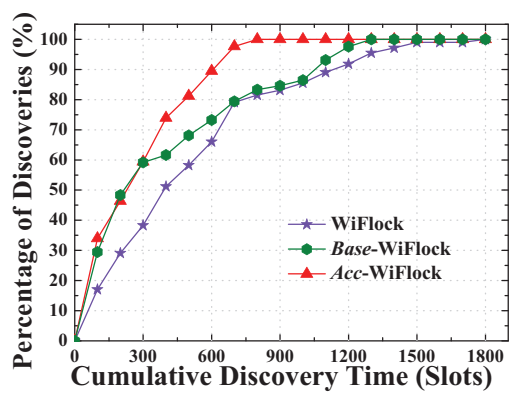

Fig. 22. WiFlock CDF.

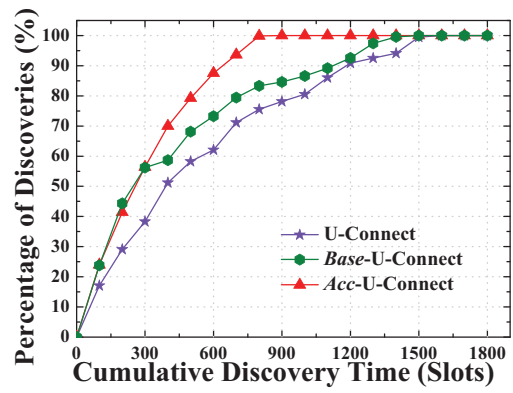

Fig. 21. U-Connect CDF.

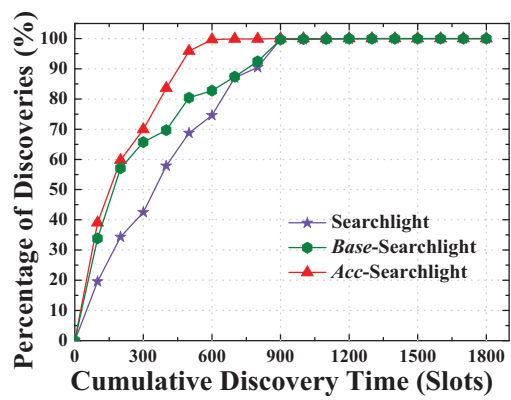

Fig. 23. Searchlight CDF.

from the point when a node is within a discovering node's rage until it was discovered by the discovering node.

\subsection{Effectiveness in Acceleration of Discovery}

In Figure 20, we plot the percentages of discoveries in terms of cumulative discovery time. In this figure, we observe that with the increase of cumulative discovery time, the percentage of discoveries also increases for all versions of Disco. Nevertheless, Acc-Disco is able to discover neighbors faster than other versions under the same duty cycle. For example, to discover more than $99 \%$ of neighbors, it takes Acc-Disco, Base-Disco, and Disco around 1,000, 1,600, and 1,700 slots, respectively. If each slot is about $10 \mathrm{~ms}$, then Acc-Disco takes a device about 10 seconds to discover more than 99\% of neighbors. This is because some nodes are not neighbors at the beginning of the experiment but become neighbors later. These results show a nearly $41.1 \%$ performance gain between Acc-Disco and Disco, which proves the value of taking known neighbors into consideration to discover unknown neighbors. Via a 37.5\% performance gain between Acc-Disco and Base-Disco, we verify the effectiveness of the temporal diversity and spatial similarity as a slot gain.

For percentage of discoveries, some nodes are not neighbors in the first place but become neighbors due to mobility. We use the cumulative time to track the percentage of actual neighbors being discovered at a certain time.

Similarly, in Figures 21, 22, and 23, we plot the same sets of curves for U-Connect, WiFlock, and Searchlight. We also observe similar performance trends as in Figure 20. For example, in Figure 21, to discover more than $99 \%$ of neighbors, the cumulative discovery time for Acc-U-Connect, Base-U-Connect, and U-Connect is around 850, 1,300, 


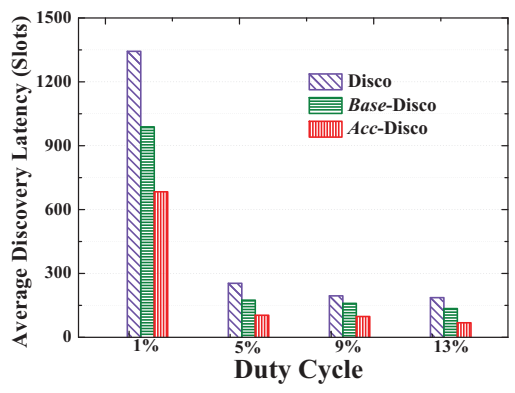

Fig. 24. Disco latency.

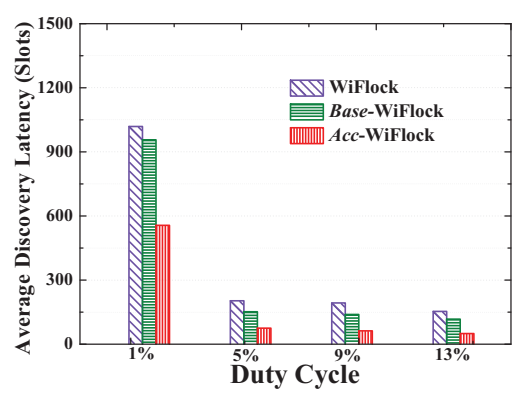

Fig. 26. WiFlock latency.

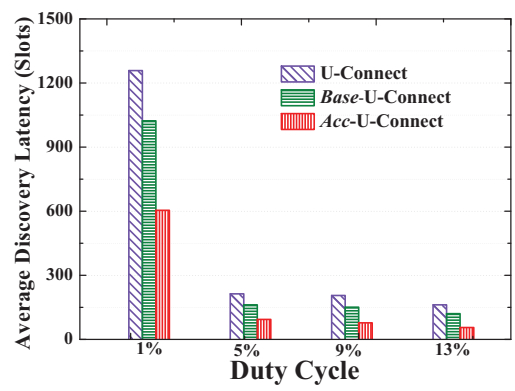

Fig. 25. U-Connect latency.

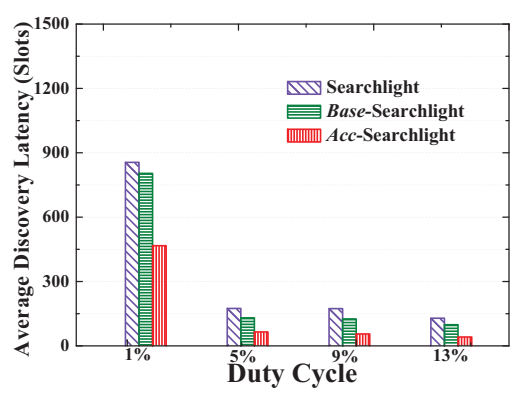

Fig. 27. Searchlight latency.

and 1,500 slots, respectively. In Figure 22, we still find a performance gain between Acc-WiFlock and other versions of WiFlock, although the performance gain of Acc in WiFlock is smaller than those in Disco and U-Connect. This is also because in WiFlock's collaboration beaconing scheme, WiFlock already employs the neighbor table to log the neighbors information, e.g., waking-up slots, duty cycle patterns, for further group maintenance. In Figure 23, we find that a performance gain between Acc-Searchlight and other versions of Searchlight becomes smaller because Searchlight significantly reduces the worst-case discovery delay.

From results in Figures 20, 21, 22, and 23, we suggest that Acc serves as an accelerating middleware for various schemes to accelerate the discovery process. Specifically, the performance gain of $A c c$ is bigger in the early stage of the discovery process.

\subsection{Impact of Duty Cycle}

In this section, we investigate the impact of a device's original duty cycle on the average discovery latency in Figures 24, 25, 26, and 27.

In all figures, we observe that with the increase of the duty cycle, the average latencies of all versions for all schemes decrease. But at each duty cycle, the versions with Acc in all four different schemes achieve the smallest latency. For example, when the duty cycle is set to $5 \%$, the average discovery latencies for Acc-Disco, Base-Disco, and Disco are around 140, 200, and 380 slots, respectively. Thus, in Disco with Acc's assistance, the average latency to discover one neighbor drops from 3.8 seconds to 1.4 seconds (at a $10 \mathrm{~ms}$ slot), a difference of $63.1 \%$. From Figures 24, 25, 26, and 27, we also observe that in general, as the duty cycle increases, the performance gain between versions with Acc and original versions also increases. For example, in Figure 24, at a $1 \%$ duty cycle, the performance gain between Acc-Disco and Disco is $50.1 \%$, whereas it increases 


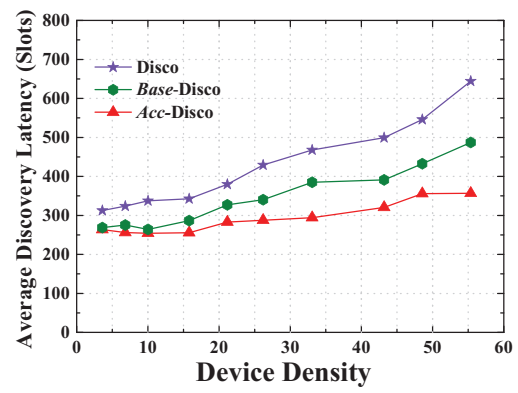

Fig. 28. Disco latency.

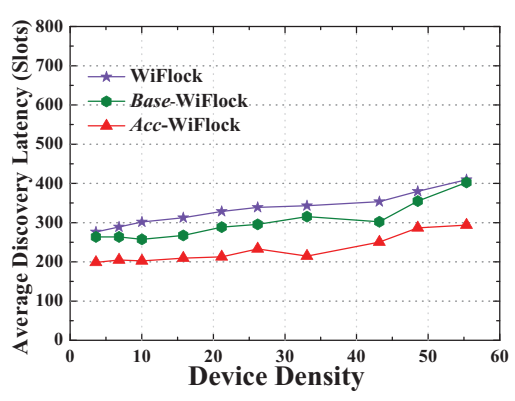

Fig. 30. WiFlock latency.

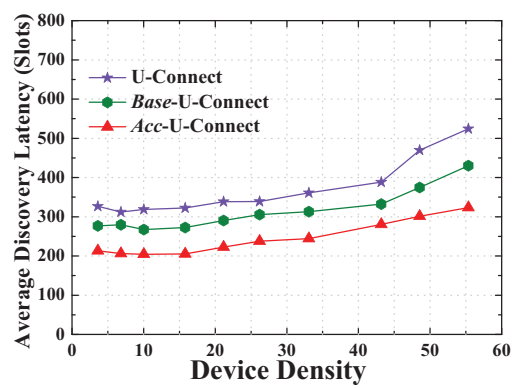

Fig. 29. U-Connect latency.

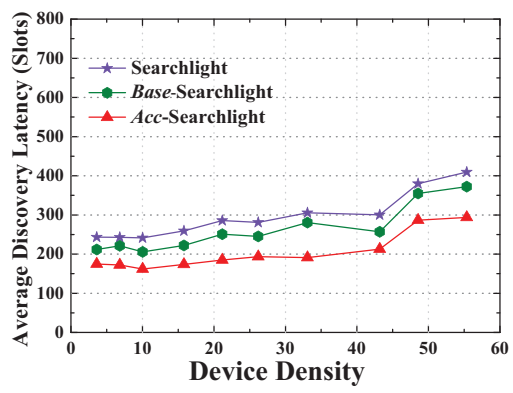

Fig. 31. Searchlight latency.

to $63.1 \%$ when the duty cycle is $5 \%$. This is because with a higher duty cycle, the devices in the network become active more frequently, leading to more neighborhood information sharing. Among other three schemes, we find that Searchlight has the best performance, which affirms our observation in Section 7.1.

Based on the preceding results, we conclude that the higher the duty cycle, the better the performance gain for Acc-assisted schemes. This is because with a higher duty cycle, a device can have more active slots that can be used by Acc for acceleration. But such acceleration effects are limited when the active slots are fewer in a lower duty cycle.

\subsection{Impact of Device Density}

In this section, we investigate the impact of the device density on the average discovery latency of four discovery schemes. The impact of device density on the average discovery latency is shown in Figures 28, 29, 30, and 31, respectively.

From all four figures, we find that as the device density increases, the average discovery latency increases for all four neighbor discovery protocols. This is because at the higher densities, the devices have more neighbors, leading to more collisions and thus more time to find all of the neighbors. When the average number of neighbors increases from 3.6 to 55.36 , the performance gain between original versions and the versions assisted with Acc also increases from $22.3 \%$ to $52.4 \%$ in Figure 28 with regard to Disco. This is because more known neighbor devices are able to share neighborhood information with discovering devices, thus accelerating the neighbor discovery process. We also observe similar results in Figures 29, 30, and 31. For example, in Figure 31, when the average number of neighbors increases from 3.6 to 55.36, the average discovery latency in Acc-Searchlight and Searchlight increases to 290 and 310 slots, respectively. 


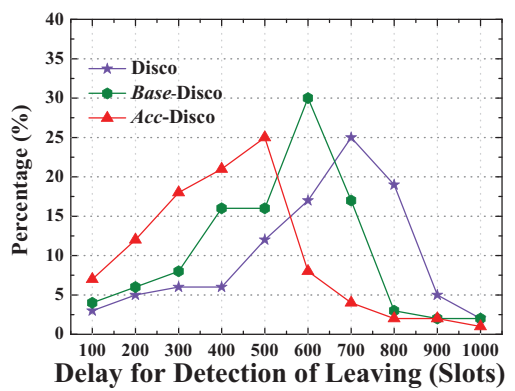

Fig. 32. Disco latency.

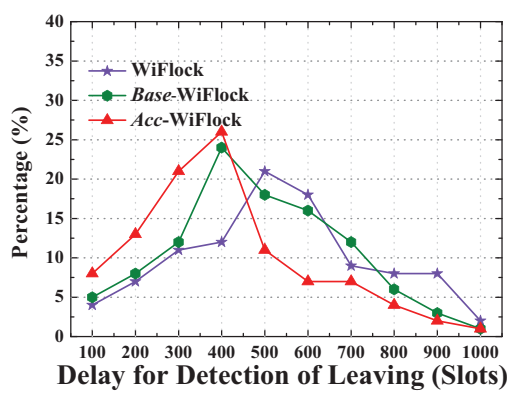

Fig. 34. WiFlock latency.

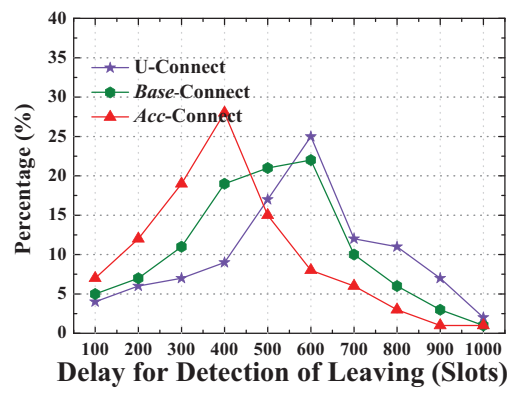

Fig. 33. U-Connect latency.

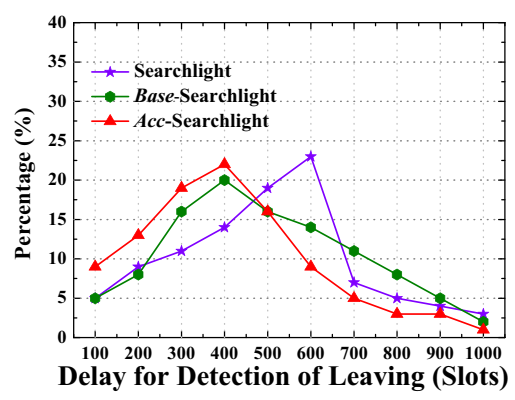

Fig. 35. Searchlight latency.

Based on the preceding results, we conclude that the higher the device density, the higher the average discovery latency. Note that even though a bigger network density can increase the collision among devices, a bigger network density also achieves a more diverse neighborhood information sharing among already known devices.

\subsection{Effectiveness in Proactive Online Rendezvous Maintenance}

In this section, we investigate the detection delay in the rendezvous maintenance mechanism in Figures 32, 33, 34, and 35. The legacy protocols, e.g., Disco [Dutta and Culler 2008], utilize the rediscovery as the rendezvous maintenance. In contrast, Acc provides an online rendezvous maintenance mechanism for them to make a discovery device $S$ to wake up at some additional slots to detect the neighbors who are believed to be leaving the radio range of $S$. In this work, we use a metric called detection delay to evaluate the performance of the online rendezvous maintenance mechanism. It is given by the time difference from the slot when a neighbor leaves the radio range of $S$ to the slot when $S$ detects that this neighbor leaves. We use a baseline design called Base to show the effectiveness of Acc. The baseline selects additional wake-up slots in random, whereas Acc utilizes the introduced binary selection. We assume that a fast maintenance is required by users, and all protocols are under the same duty cycle for a fair comparison. Due to its proactive online rendezvous maintenance, the protocols assisted by Acc would have a better performance than the original protocols and Base-assisted protocols.

The distributions of the detection delay regarding Acc on different protocols are given in Figures 32, 33, 34, and 35, respectively. We find that the Acc-assisted protocols have the lowest average detection delay, which indicates that with the help of Acc, a neighbor discovery protocol can quickly detect the leaving of a neighbor, thanks to the common 
neighbor-based maintenance mechanism. Further, Acc-assisted protocols have a better performance than Base-assisted protocols, because Acc's binary selection is more effective than the random selection of Base. Based on the results, we conclude that Acc's proactive online rendezvous maintenance mechanism assists existing neighbor discovery in reducing the detection delay of the leaving of neighbors.

\section{CROWD-ALERT APPLICATION}

In this section, to prove the real-world value of Acc, we propose and evaluate a CrowdAlert application with which taxi drivers can quickly navigate optimal directions to travel to maximize the possibility of picking up passengers by an Acc-assisted neighbor discovery after they drop off passengers, i.e., a faster neighbor discovery is demanded.

Based on the current setting of taxicab systems in metropolitan areas, every taxi has a wireless communication interface (WiFi or WiMax) installed to send its locations and status (with or without passengers) back to base stations for accounting purposes. Thus, we envision that a taxicab would primarily use its radio in the infrastructure mode, as it has to communicate with a base station frequently for accounting. Only during gaps between communication with base stations can a taxi set its radio to the ad hoc mode for peer-to-peer neighbor discovery. Thus, it leads to a duty cycle between the infrastructure and ad hoc mode not for energy but for radio usage. Based on the broadcast status of nearby taxis, a taxi driver can obtain the crowd levels both in terms of the number of taxis and number of passengers in a given area. Taxi drivers who install this application can form groups of common interest to optimize their profits. Individual drivers using this application can quickly navigate to areas with a low density of taxis (and presumably a high passenger density) to maximize pickups (and thus profits).

Our proposed protocol, Acc, provides a mechanism for distributed discovery of neighbors in an accelerated manner, which we will adapt to the application installed on the taxi. We will describe our application in further detail and evaluate the efficiency of this scheme in discovering neighbors in a timely fashion.

\subsection{Application Background}

In our application, each taxicab broadcasts its own status record, i.e., date and time, availability, direction, GPS coordinates, to its neighboring taxis during the time it is not communicating with base stations. The broadcast is performed based on a concrete discovery scheme, e.g., Disco. According to the information collected, when a taxi becomes available and the driver wants to quickly pick up a passenger, i.e., an on-demand acceleration is required, the taxi driver can navigate to the optimal directions, as determined by the number of nearby competing taxis and nearby potential passengers. These two metrics can maximize the probability of picking up the next nearby passengers.

Generally, the fewer the competing taxis, the higher the probability of picking up passengers. With distributedly collected status records about neighboring taxis, our Crowd-Alert computes the location distribution of competing taxis that also aim to pick up new passengers. Similarly, the more the potential passengers, the higher the probability of picking up. Without the active participation of passengers, however, it is unrealistic to expect to obtain such a distribution based on taxi status records alone. But we can obtain a cumulative location distribution of passengers who have just entered or exited taxis, i.e., served passengers, by observing the change of the availability bit (from 0 to 1 or from 1 to 0 ) in two consecutive status records about the same taxi. Further, we assume that a location distribution of served passengers is an indication of that of potential passengers, but how to obtain a distribution based on the indication is outside the scope of this article. To focus on system levels, we simply utilize the location distribution of served passengers as that of potential passengers. 


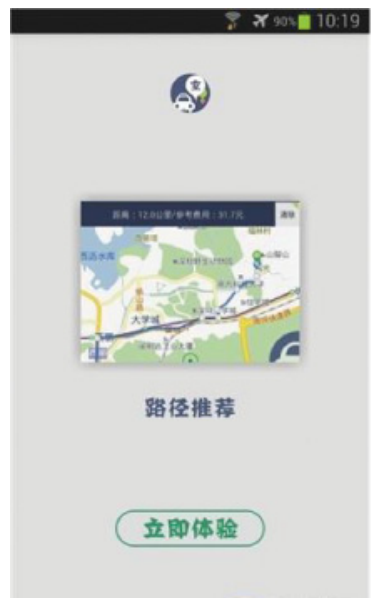

Finding a Route

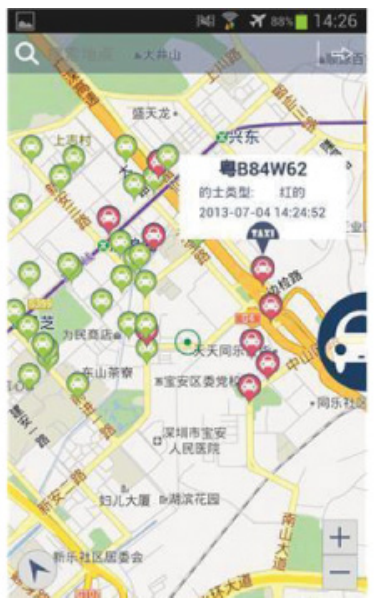

Competing Taxicabs

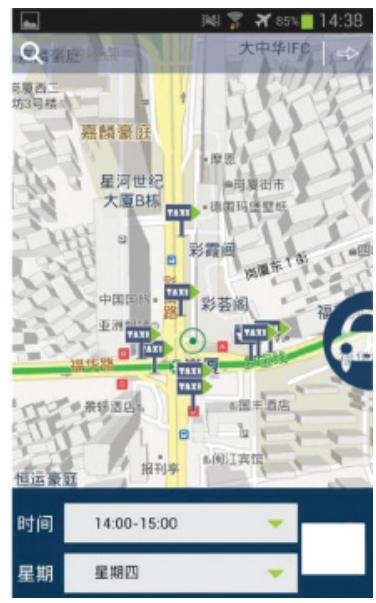

Potential Passengers

Fig. 36. App screenshots.

\begin{tabular}{|c|c|}
\hline \multicolumn{2}{|c|}{ Taxicab Network Summary } \\
\hline Collection Period & 6 Months \\
\hline Collection Date & $01 / 01 / 12-06 / 30 / 12$ \\
\hline Numbe of Taxicabs & 14,453 \\
\hline Number of Passengers & $98,472,628$ \\
\hline Total Travel Distance & $594,031,428(\mathrm{KM})$ \\
\hline Total Fare & $2,255,052,932(\mathrm{CNY})$ \\
\hline Average Travel Distance & $6.032(\mathrm{KM})$ \\
\hline Average Fare & $22.9(\mathrm{CNY})$ \\
\hline
\end{tabular}

Fig. 37. Statistics.

Based on the distributions of competing taxis and served passengers, Crowd-Alert can maximize the possibility of picking up passengers by guiding a taxi to a direction with fewer competing taxis or more served passengers. A faster discovery achieved by our Acc can assist a navigating scheme in making a timely decision.

\subsection{Application Evaluation}

We embed the Crowd-Alert function into one of our taxicab-booking apps for the taxicab network in Shenzhen. In Figure 36, we show the app screenshots for finding a route, checking nearby taxicabs, and potential passengers.

But due to privacy reasons and limited installations, we cannot evaluate Crowd-Alert based on the data from the app in large scales. Instead, we evaluate the Crowd-Alert application using a real-world dataset collected from taxis in Shenzhen over 6 months. We first introduce the dataset and then present the evaluation results in terms of reduction of discovery latency and accelerating the navigation.

8.2.1. Dataset. The dataset consists of 6 months of GPS traces from 14,453 taxis. The data is used by the government for the urban transportation pattern search. Each taxi uploads its records every 15 to 30 seconds, with each record consisting of the following parameters: (i) plate number; (ii) date and time; (iii) GPS coordinates; and (iv) availability, whether or not a passenger is in this taxi when the record is uploaded. Figure 37 summarizes details of the used datasets. Based on the preceding GPS trace 


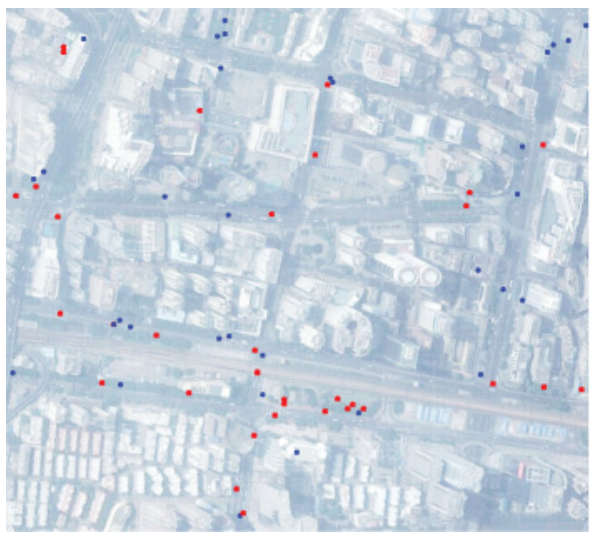

Fig. 38. Distribution of competing taxis.

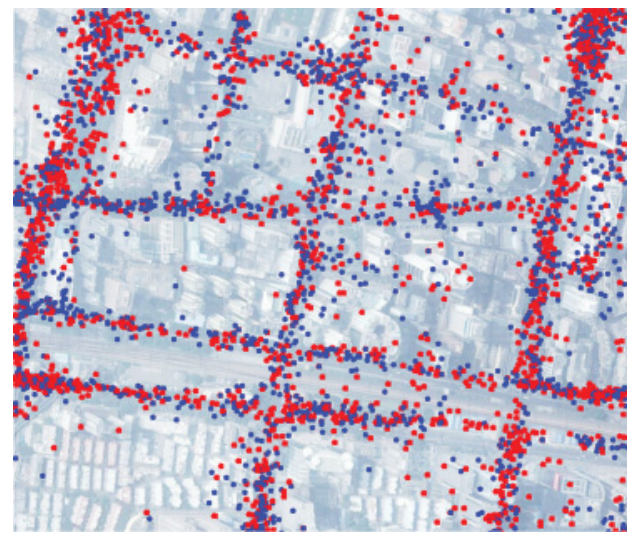

Fig. 39. Distribution of served passengers.

records, we can obtain a location distribution of competing taxis or served passengers, as shown in Figures 38 and 39.

Figure 38 shows a taxi distribution of an area about 1 square kilometer (GPS coordinates $X X X .538-X X X .547 \times X X X .108-X X X .117)$ based on a 10 s uploading window in the rush hour of 1 day, i.e., 5PM. Red points indicate the taxis with passengers, and blue points indicate the taxis without passengers. Figure 39 shows a served passenger distribution in the same area as in Figure 38 in a 2-hour uploading time window in 1 day, i.e., from $4 \mathrm{PM}$ to $6 \mathrm{PM}$. Red points indicate the locations of passengers entering taxis, and blue points indicate the locations of passengers exiting taxis.

8.2.2. Reduction of Discovery Latency. Before we investigate the effects of Acc's accelerations on the navigation of taxis, we first perform a trace-driven simulation on the dataset to verify how Acc accelerates discovery in this taxi network. With a total duty cycle of $\frac{4}{30}$, we compare four schemes-Disco, U-connect, WiFlock, and Searchlightwith and without the assistance of Acc. This duty cycle rate is decided by the fact that a taxi has to communicate with base stations about accounting 26 seconds per 30 seconds. The rest of the time can be used for peer-to-peer neighbor discovery based on the ad hoc mode. We assume that the taxi is equipped with a radio that has a large communication radius and that a communication range of $3 \mathrm{~km}$ is used. A smaller communication radius, e.g., $100 \mathrm{~m}$ in a WiFi interface, does not allow our system to fully exploit the quick discovery scheme in a taxi network. This is because a $100 \mathrm{~m}$ communication radius is too small for vehicular networks where even the length of a static taxi is about $5 \mathrm{~m}$. Therefore, navigation based on such a small communication radius will lead to an extremely low density of taxis and may not have any obvious performance difference under various discovery schemes.

From Figure 40, we observe that Acc-Disco is able to discover neighboring taxis faster than Disco under the same duty cycle. For example, to discover all neighboring taxis, it takes Acc-Disco and Disco around 7 minutes and 9 minutes, respectively. These results show a $22.2 \%$ performance gain between Acc-Disco and Disco. We also observe that the performance gain achieves the maximum in the first half of the discovery process, where a taxi can detect more than half of its neighboring taxis within 3 minutes. This suggests that $A c c$ can enable $A c c$-Disco to quickly find the most neighbor taxis in a very short period of time, which can assist a driver to more quickly drive in the optimal directions. Similarly, in Figures 41, 42, and 43, we plot the same sets of curves for U-Connect, WiFlock, and Searchlight. In Figures 41, 42, and 43, we observe similar 


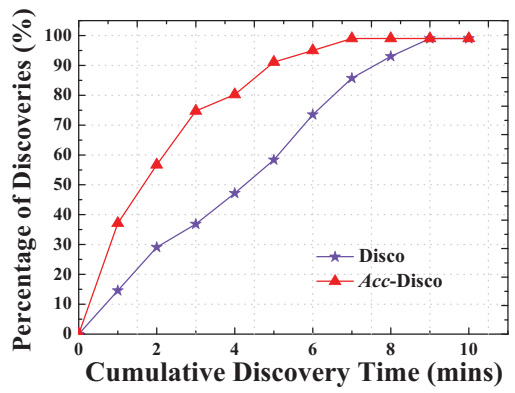

Fig. 40. Disco latency.

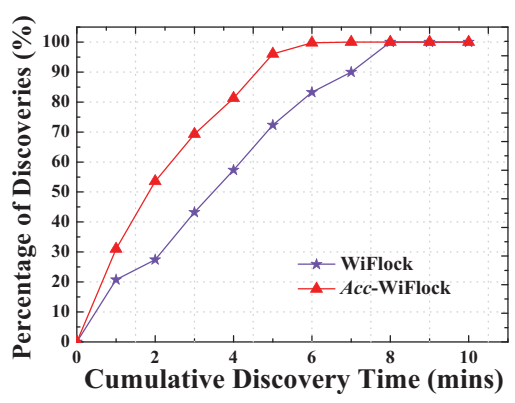

Fig. 42. WiFlock latency.

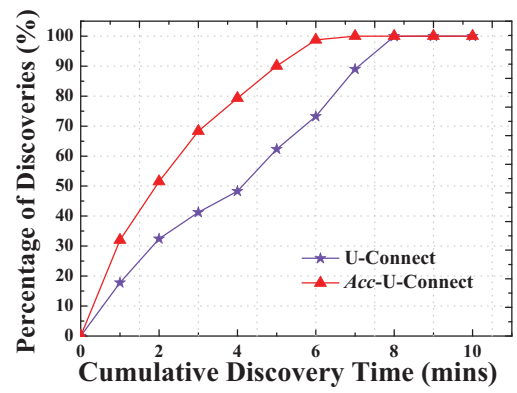

Fig. 41. U-Connect latency.

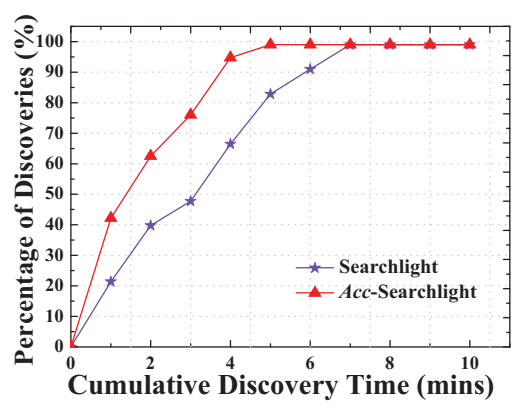

Fig. 43. Searchlight latency.

performance trends between Acc-assisted versions and original versions. For example, in Figure 41, to discover all neighboring taxis, the cumulative discovery time for UConnect and Acc-U-Connect is around 6 minutes and 8 minutes, respectively, achieving a $25 \%$ performance gain. In Figures 42 and 43, we still observe a performance gain between Acc-WiFlock and WiFlock and between Acc-Searchlight and Searchlight.

From the results in Figures 40, 41, 42, and 43, we conclude that Acc can accelerate various discovery schemes in this taxi network and may serve as an augmenting layer to accelerate discovery to quickly navigate taxis to optimal directions.

8.2.3. Acceleration of Navigation. In this section, we evaluate the performance of Acc in accelerating the navigation for taxis in Crowd-Alert. With a total duty cycle of $\frac{4}{30}$, we compare three navigating results based on different discovery results of discovery schemes:

(i) Navigating with Disco: Navigating taxis with the results of Disco;

(ii) Navigating with Acc-Disco: Navigating taxis with the results of Acc-Disco; and

(iii) Navigating with Oracle: Navigating taxis with the results of an Oracle discovery scheme where a taxi can instantly know these two distributions without delay.

Under all navigations, a taxi has the same preferable directions for fewer competing taxis, more served passengers, or a ratio between them. But since the employed discovery schemes are different, a navigation with a faster discovery scheme may achieve better performance. The performance is characterized by three metrics: competing taxis density, served passengers density, and a ratio between them. A faster discovery may assist a navigation scheme in quickly navigating taxis to an area with fewer competing taxis or more served passengers. 


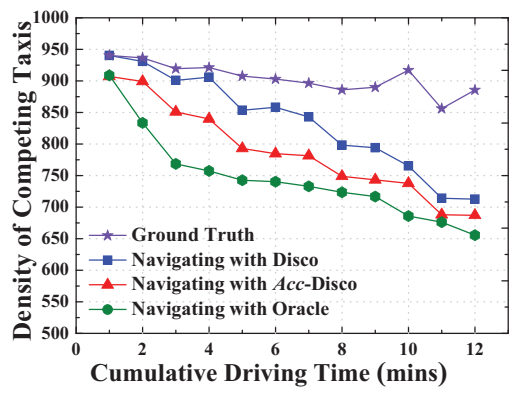

Fig. 44. Density in one smart taxi.

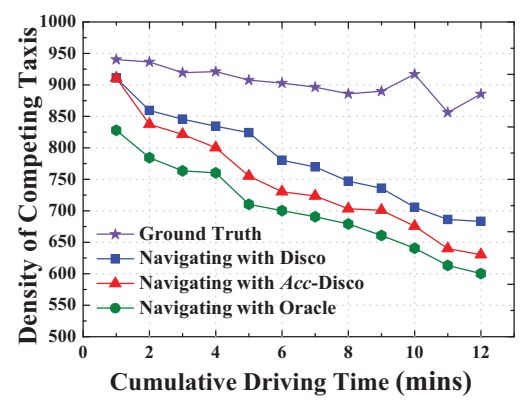

Fig. 45. Density in $10 \%$ of smart taxis.

To show the difference with or without our application, we also compare the preceding three schemes with Ground Truth without navigation, where the density is computed based on original taxi traces without altering the routes of any taxis. Note that given the density of competing taxis or served passengers, how to select the optimal route to achieve the optimal density is outside the scope of this article. We simply let taxis greedily select one out of four directions in an intersection according to densities in every direction and then compute densities of competing taxis or served passengers in its neighborhood every minute. We compare the performance of Acc under two conditions: only one smart taxi using navigation strategies and $10 \%$ of total taxis using them.

(a) Density of Competing Taxis. We investigate the densities of competing taxis in three different navigating strategies. We report the results of navigating only one taxi or $10 \%$ of total taxis to select a direction with a lower density of competing taxis, using a $3 \mathrm{~km}$ communication radius in Figures 44 and 45 , respectively.

Only one smart taxi. We show the situation where one smart driver uses our app to find the optimal route. In Figure 44, as more driving time is allowed, there exists a jitter in the density of competing taxis of Ground Truth, which has no tendency toward consistent increases or decreases, whereas those of Disco, AccDisco, and Oracle decrease. This is because the taxis with Disco, Acc-Disco, and Oracle navigate to a direction with fewer competing taxis, so after 3 minutes the density of competing taxis within its range drops. Compared to Ground Truth, after 12 minutes, under Disco the density decreases about $16 \%$ and under Acc-Disco the density decreases about 25\%, whereas Oracle outperforms Disco and Acc-Disco in all cumulative driving times with a maximal performance gain of $5.1 \%$ and $10.1 \%$, respectively. From the preceding results, Oracle does not significantly outperform Disco and Acc-Disco. One possible reason for this phenomenon is that the beginning time and location for this one smart taxi is the rush hour in a downtown area. Therefore, even though Oracle provides the local optimal direction to reduce the density of competing taxis, the effect is limited.

$10 \%$ smart taxis. We show the situation where taxi drivers from one company (accounting for $10 \%$ of taxicabs) use our app to find the optimal route. Figure 45 plots results of $10 \%$ of total taxis using our application. Compared to the results in Figure 44, all strategies have better performance, except for Ground Truth, which remains the same. This is because the more the taxis use our applications, the more the taxis will select the direction with lower taxi density, which in turn will achieve a more uniform taxi distribution. Comparing Figures 44 and 45, we note that the performance gain between navigating with Acc-Disco and Disco increases from $25 \%$ to $29 \%$, indicating that Acc-Disco is more efficient when more taxis use our application. 


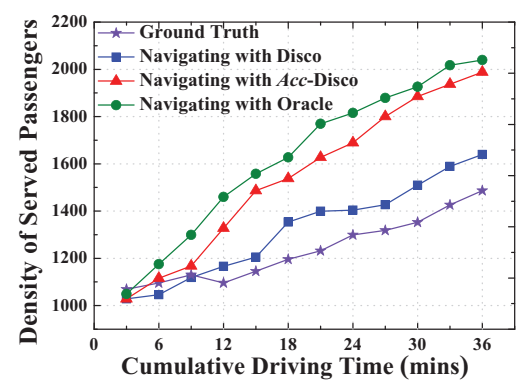

Fig. 46. Density of passengers.

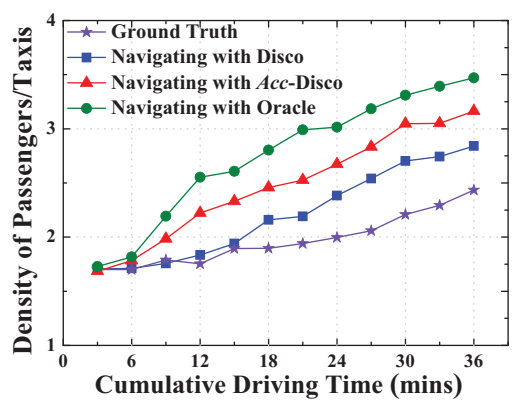

Fig. 47. Density of passengers/taxis.

(b) Density of Served Passengers. We show the effectiveness of Acc in assisting the navigating scheme to navigate taxis to a direction with more served passengers in Figure 46. Since the percentage of taxis using our application is not directly relevant to the density of already served passengers, we only show the results on the $10 \%$ of smart taxis scenario. As in Figures 38 and 39, the density of served passengers is denser than densities of competing taxis, so we use a $0.5 \mathrm{~km}$ radius to compute the density. Figure 46 plots the comparisons of cumulative densities of the served passengers. With an increase in the cumulative driving time, the cumulative density of served passengers in a taxi's neighborhood also generally increases for all schemes. The reason for the increases in navigation under Disco, Acc-Disco, or Oracle is obvious, because that is the objective of our application. But the reason for the increase in Ground Truth is not so obvious. A possible explanation is that taxi drivers have rich experiences that help them select the area to maximize the probability of picking up. The location of already-served passengers offers a strong indication to the location of potential passengers. Therefore, even without our application, experienced taxi drivers will still go to the area with more served passengers. But compared to Ground Truth, Disco can assist taxi drivers in finding the optimal direction more quickly via discovery. Therefore, there is a performance gain between Disco and Ground Truth with a maximum of $10 \%$ after 36 minutes. In contrast, navigation with Acc-Disco is able to discover neighbors even faster than that with Disco. For example, it outperforms those under Ground Truth and Disco with maximal gains of $21 \%$ and $35 \%$, respectively, but has a worse performance than Oracle. Therefore, we conclude that with Acc-Disco, a navigation scheme can more quickly guide the taxi to a direction with a high density of served passengers.

(c) Density of Served Passengers and Competing Taxis. Built upon the two previous sections, we investigate the effectiveness of Acc in assisting navigating schemes in leading taxis to a direction with more served passengers and fewer competing taxis at the same time. Thus, we use a ratio equal to the number of served passengers and competing taxis as a new metric for the navigation instead of considering served passengers and competing taxis separately. In Figure 47, with an increase in the cumulative driving time, the ratio between the density of passengers and the density of competing taxis in a taxi's neighborhood also generally increases for all schemes. Similar to Figure 46, we find that compared to Disco and Ground Truth, Acc-Disco or Oracle always more quickly navigates the taxicabs to a direction with both more passengers and fewer competing taxis. The performance gains among all four schemes are similar to those in Figure 47. 


\section{CONCLUSION}

In this work, we analyze, design, implement, and evaluate Acc, an augmenting layer for the acceleration of neighbor discovery in existing deterministic discovery schemes. Our technical endeavors provide a few valuable insights, which are hoped to be useful to realize $A c c$-based on-demand neighbor discovery applications in various domains. Specifically, (i) we found that known neighbors can help a device learn unknown neighbors indirectly, which is the key insight about our on-demand discovery; (ii) we designed Acc as an independent middleware in the networking architecture without merging it into existing neighbor discovery protocols, which makes Acc a transparent accelerator without the dependence on the preceding applications or the following neighbor discovery protocols; (iii) we characterized a slot with a novel concept called temporal-spatial coverage to indicate the utility of a discovery device to wake up in the slot, and this characterization is fully distributed and values the slots with neighbors having higher temporal diversity and larger spatial similarity; and (iv) we designed a real-world taxi application where $A c c$ is used to accelerate the process of taxicab drivers finding efficient routes, which serves as a real-world example to justify the motivation behind the design of Acc.

\section{APPENDIX: PROOF OF COMPETITIVE RATIO}

We analyze the performance of our scheduling by comparing it to its Oracle version with a complete neighbor table $N^{(S, S)}$. In our scheduling, a device $S$ 's neighbor table of in slot $t_{0}$, denoted as $n_{t_{0}}^{(S, S)}$, is processed piece by piece. This is a classic nature of an online algorithm, which processes its incomplete input piece by piece from the start. Because of this incomplete input, an online algorithm is forced to make suboptimal decisions. To study this suboptimality, a competitive analysis is proposed to compare the relative performance of an online algorithm to its Oracle version that has a complete input. An online algorithm is competitive if its competitive ratio $\rho$, an performance ratio between it and its Oracle version, is bounded. To obtain $\rho$, we utilize qualities of selected active slots to represent algorithms' performances, indicating how much new neighbor information can be collected in these slots. The qualities of these slots can be represented by slot gains. Therefore, we can analyze $\rho$ by comparing the slot gains under our online scheduling and its Oracle version, employing different neighbor tables. In the following, we prove that $\rho$ is bounded by a parameter $R$, which is the size ratio between $n_{t_{0}}^{(S, S)}$ and $N^{(S, S)}$.

Assumptions are as follows: (i) in slot $t_{0}$, a device $S$ has already discovered a portion of its neighbors in $n_{t_{0}}^{(S, S)}$; (ii) a parameter $R=\frac{\left|n_{t}^{(S, S)}\right|}{\left|N^{(S, S)}\right|}<1$ is given, which is the ratio between the number of neighbors in $n_{t_{0}}^{(S, S)}$ and $N^{(S, S)}$; (iii) all discovered neighbors are uniformly distributed in $N^{(S, S)}$; and (iv) to minimize the effect of duty cycles, duty cycle patterns for different devices are the same.

Via Equation (3) and assumption (i), $\rho$ is given by

$$
\frac{1}{\rho}=\frac{\gamma_{t_{0} \rightarrow t}^{(S)}(\text { Oracle })}{\gamma_{t_{0} \rightarrow t}^{(S)}(\text { Online })}=\frac{\sum_{i \in N^{(S, S)}} \alpha_{t_{0} \rightarrow t}^{(i, S)} \beta_{t_{0}}^{(i, S)}}{\sum_{j \in n_{t_{0}}^{(S, S)}} \alpha_{t_{0} \rightarrow t}^{(j, S)} \bar{\beta}_{t_{0}}^{(j, S)}},
$$

where $\alpha_{t_{0} \rightarrow t}^{(i, S)}$ and $\alpha_{t_{0} \rightarrow t}^{(j, S)}$ is temporal diversity for device $i \in N^{(S, S)}$ and $j \in n_{t_{0}}^{(S, S)}$, respectively, and $\beta_{t_{0}}^{(i, S)}$ and $\bar{\beta}_{t_{0}}^{(j, S)}$ is spatial similarity for device $i \in N^{(S, S)}$ and $j \in n_{t_{0}}^{(S, S)}$, 
respectively. Equation (5) can be reorganized as follows:

$$
\frac{1}{\rho}=\frac{\sum_{j \in n_{t_{0}}^{(S, S)}} \alpha_{t_{0} \rightarrow t}^{(j, S)} \beta_{t_{0}}^{(j, S)}}{\sum_{j \in n_{t_{0}}^{(S, S)}} \alpha_{t_{0} \rightarrow t}^{(j, S)} \bar{\beta}_{t_{0}}^{(j, S)}}+\frac{\sum_{i \in n_{t_{0}}^{(S, S)}} \alpha_{t_{0} \rightarrow t}^{(i, S)} \beta_{t_{0}}^{(i, S)}}{\sum_{j \in n_{t_{0}}^{(S, S)}} \alpha_{t_{0} \rightarrow t}^{(j, S)} \bar{\beta}_{t_{0}}^{(j, S)}},
$$

where $\overline{n_{t_{0}}^{(S, S)}}$ is the complement of $n_{t_{0}}^{(S, S)}$, given $N^{(S, S)}$.

The following analyzes the first term in Equation (6). According to Equation (2) and assumption (ii), we have

$$
\frac{\beta_{t_{0}}^{(j, S)}}{\bar{\beta}_{t_{0}}^{(j, S)}}=\frac{\left|N_{t_{0}}^{(j, S)}\right| /\left|N_{t_{0}}^{(S, S)}\right|}{\left|n_{t_{0}}^{(j, S)}\right| /\left|n_{t_{0}}^{(S, S)}\right|}=\frac{\left|N_{t_{0}}^{(j, S)}\right|}{\left|n_{t_{0}}^{(j, S)}\right|} \frac{\left|n_{t_{0}}^{(S, S)}\right|}{\left|N_{t_{0}}^{(S, S)}\right|}=\frac{R}{R^{\prime}},
$$

where $R^{\prime}=\frac{\left|n_{t_{0}}^{(j, S)}\right|}{\left|N_{t_{0}}^{(j, S)}\right|}<1$. Therefore, the first term in Equation (6) can be represented as follows:

$$
\frac{\sum_{j \in n_{t_{0}}^{(S, S)}} \alpha_{t_{0} \rightarrow t}^{(j, S)} \beta_{t_{0}}^{(j, S)}}{\sum_{j \in n_{t_{0}}^{(S, S)}} \alpha_{t_{0} \rightarrow t}^{(j, S)} \bar{\beta}_{t_{0}}^{(j, S)}}=\frac{\frac{R}{R^{\prime}} \sum_{j \in n_{t_{0}}^{(S, S)}} \alpha_{t_{0} \rightarrow t}^{(j, S)} \bar{\beta}_{t_{0}}^{(j, S)}}{\sum_{j \in n_{t_{0}}^{(S, S)}} \alpha_{t_{0} \rightarrow t}^{(j, S)} \bar{\beta}_{t_{0}}^{(j, S)}}=\frac{R}{R^{\prime}}>1,
$$

where $R>R^{\prime}$ because, due to assumption (iii), not all $i \in \overline{n_{t_{0}}^{(j, S)}}$ are neighbors of $j$.

The following analyzes the second term of Equation (6). Due to assumption (iv), $\forall i$, $j \in N^{(S, S)}, \alpha_{t_{0} \rightarrow t}^{(i, S)}=\alpha_{t_{0} \rightarrow t}^{(j, S)}$. Thus, the second term in Equation (6) can be reorganized as follows:

$$
\frac{\sum_{i \in n_{t_{0}}^{(S, S)}} \alpha_{t_{0} \rightarrow t}^{(i, S)} \beta_{t_{0}}^{(i, S)}}{\sum_{j \in n_{t_{0}}^{(S, S)}} \alpha_{t_{0} \rightarrow t}^{(j, S)} \bar{\beta}_{t_{0}}^{(j, S)}}=\frac{\alpha_{t_{0} \rightarrow t}^{(i, S)} \sum_{i \in n_{t_{0}}^{(S, S)}} \beta_{t_{0}}^{(i, S)}}{\alpha_{t_{0} \rightarrow t}^{(j, S)} \sum_{j \in n_{t_{0}}^{(S, S)}} \bar{\beta}_{t_{0}}^{(j, S)}}=\frac{\sum_{i \in n_{t_{0}}} \overline{n_{\text {S.S }}} \beta_{t_{0}}^{(i, S)}}{\sum_{j \in n_{t_{0}}^{(S, S)}} \bar{\beta}_{t_{0}}^{(j, S)}}
$$

Based on assumption (iii), $\forall j \in n_{t_{0}}^{(S, S)}$ and $\forall i \in \overline{n_{t_{0}}^{(S, S)}}$ are randomly and uniformly distributed in $N^{(S, S)}$. Therefore, $\forall i, j \in N^{(S, S)}, \beta_{t_{0}}^{(i, S)}=\beta_{t_{0}}^{(j, S)}$, and $\forall i, j \in n_{t_{0}}^{(S, S)}, \bar{\beta}_{t_{0}}^{(i, S)}=$ $\bar{\beta}_{t_{0}}^{(j, S)}$. Thus,

$$
\frac{\sum_{i \in n_{t_{0}}^{(S, S)}} \beta_{t_{0}}^{(i, S)}}{\sum_{j \in n_{t_{0}}^{(S, S)}} \bar{\beta}_{t_{0}}^{(j, S)}}=\frac{\overline{\mid n_{t_{0}}^{(S, S)}} \mid \beta_{t_{0}}^{(i, S)}}{\left|n_{t_{0}}^{(S, S)}\right| \bar{\beta}_{t_{0}}^{(j, S)}}
$$

Because $\overline{n_{t_{0}}^{(S, S)}} \cup n_{t_{0}}^{(S, S)}=N^{(S, S)}$ and $\frac{\left|t_{t_{0}}^{(S, S)}\right|}{\left|N^{(S, S)}\right|}=R$, we have $\left|\overline{n_{t_{0}}^{(S, S)}}\right|=\frac{1-R}{R}\left|n_{t_{0}}^{(S, S)}\right|$. Therefore, Equation (10) can be rewritten as follows:

$$
\frac{\left|\overline{n_{t_{0}}^{(S, S)}}\right| \beta_{t_{0}}^{(i, S)}}{\left|n_{t_{0}}^{(S, S)}\right| \bar{\beta}_{t_{0}}^{(j, S)}}=\frac{\frac{1-R}{R}\left|n_{t_{0}}^{(S, S)}\right| \beta_{t_{0}}^{(i, S)}}{\left|n_{t_{0}}^{(S, S)}\right| \bar{\beta}_{t_{0}}^{(j, S)}}=\frac{1-R}{R} \frac{\beta_{t_{0}}^{(i, S)}}{\bar{\beta}_{t_{0}}^{(j, S)}} .
$$

Since $i$ and $j$ are two arbitrary devices in the networks, based on the analysis of Equation (7), $\frac{\beta_{t_{0}}^{(i, S)}}{\bar{\beta}_{t_{0}}^{(j, S)}}>1$. Therefore, we have

$$
\frac{1-R}{R} \frac{\beta_{t_{0}}^{(i, S)}}{\bar{\beta}_{t_{0}}^{(j, S)}}>\frac{1-R}{R}=\frac{1}{R}-1
$$


Based on Equation (8), we have the first term in Equation (6); based on Equations (9) through (12), we have the second term in Equation (6). Therefore, Equation (6) can be rewritten as follows:

$$
\frac{1}{\rho}=\frac{R}{R^{\prime}}+\frac{1-R}{R} \frac{\beta_{t_{0}}^{(i, S)}}{\bar{\beta}_{t_{0}}^{(j, S)}}>1+\frac{1}{R}-1=\frac{1}{R} .
$$

Finally, we have the competitive ratio $\rho$ :

$$
\rho=\frac{\gamma_{t_{0} \rightarrow t}^{(S)}(\text { Online })}{\gamma_{t_{0} \rightarrow t}^{(S)}(\text { Oracle })}<R .
$$

According to the preceding analysis, we have obtained the competitive ratio $\rho$ of our online scheduling algorithm.

\section{REFERENCES}

Denizhan N. Alparslan and Khosrow Sohraby. 2007. Two-dimensional modeling and analysis of generalized random mobility models for wireless ad hoc networks. IEEE/ACM Transactions on Networking 15, 3, 616-629. DOI : http://dx.doi.org/10.1109/TNET.2007.893873

Mehedi Bakht, Matt Trower, and Robin Hilary Kravets. 2012. Searchlight: Won't you be my neighbor? In Proceedings of the 18th Annual International Conference on Mobile Computing and Networking (Mobicom'12). ACM, New York, NY, 185-196. DOI : http://dx.doi.org/10.1145/2348543.2348568

James Biagioni, Tomas Gerlich, Timothy Merrifield, and Jakob Eriksson. 2011. EasyTracker: Automatic transit tracking, mapping, and arrival time prediction using smartphones. In Proceedings of the 9th ACM Conference on Embedded Networked Sensor Systems (SenSys'11). ACM, New York, NY, 68-81. DOI : http://dx.doi.org/10.1145/2070942.2070950

Prabal Dutta, Paul M. Aoki, Neil Kumar, Alan Mainwaring, Chris Myers, Wesley Willett, and Allison Woodruff. 2009. Common sense: Participatory urban sensing using a network of handheld air quality monitors. In Proceedings of the 7th ACM Conference on Embedded Networked Sensor Systems (SenSys'09). ACM, New York, NY, 349-350. DOI:http://dx.doi.org/10.1145/1644038.1644095

Prabal Dutta and David Culler. 2008. Practical asynchronous neighbor discovery and rendezvous for mobile sensing applications. In Proceedings of the 6th ACM Conference on Embedded Network Sensor Systems (SenSys'08). ACM, New York, NY, 71-84. DOI :http://dx.doi.org/10.1145/1460412.1460420

Prabal Dutta and Lakshminarayanan Subramanian. 2010. Human-enabled microscopic environmental mobile sensing and feedback. In Proceedings of the AAAI Spring Symposium: Artificial Intelligence for Development. http://dblp.uni-trier.de/db/conf/aaaiss/aaaiss2010-1.html.

Jeremy Elson and Kay Römer. 2003. Wireless sensor networks: A new regime for time synchronization. ACM SIGCOMM Computer Communication Review 33, 1, 149-154. DOI:http://dx.doi.org/10. $1145 / 774763.774787$

Facebook. 2013. Facebook Places. Retrieved October 26, 2015, from https://www.facebook.com/places/.

Foursquare. 2013. Foursquare Home Page. Retrieved October 26, 2015, from http://www.foursquare.com.

Raghu K. Ganti, Fan Ye, and Hui Lei. 2011. Mobile crowdsensing: Current state and future challenges. IEEE Communications Magazine 49, 11, 32-39.

Google. 2013. Google Latitude. Retrieved October 26, 2015, from http://www.google.com/latitude.

Jyh-How Huang, Saqib Amjad, and Shivakant Mishra. 2005. CenWits: A sensor-based loosely coupled search and rescue system using witnesses. In Proceedings of the 3rd ACM Conference on Embedded Networked Sensor Systems (SenSys'05).

Hyewon Jun, Mostafa H. Ammar, Mark D. Corner, and Ellen W. Zegura. 2006. Hierarchical power management in disruption tolerant networks with traffic-aware optimization. In Proceedings of the 2006 SIGCOMM Workshop on Challenged Networks (CHANTS'06). ACM, New York, NY, 245-252. DOI: http://dx. doi.org/10.1145/1162654.1162662

Arvind Kandhalu, Karthik Lakshmanan, and Ragunathan (Raj) Rajkumar. 2010. U-connect: A low-latency energy-efficient asynchronous neighbor discovery protocol. In Proceedings of the 9th ACM/IEEE International Conference on Information Processing in Sensor Networks (IPSN'10). ACM, New York, NY, 350-361. DOI :http://dx.doi.org/10.1145/1791212.1791253 
Shouwen Lai, B. Ravindran, and Hyeonjoong Cho. 2010. Heterogenous quorum-based wake-up scheduling in wireless sensor networks. IEEE Transactions on Computers 59, 11, 1562-1575.

Nicholas D. Lane, Emiliano Miluzzo, Hong Lu, Daniel Peebles, Tanzeem Choudhury, and Andrew T. Campbell. 2010. A survey of mobile phone sensing. IEEE Communications Magazine 48, 9, 140-150.

Hengchang Liu, Jingyuan Li, Zhiheng Xie, Shan Lin, Kamin Whitehouse, John A. Stankovic, and David Siu. 2010. Automatic and robust breadcrumb system deployment for indoor firefighter applications. In Proceedings of the 8th International Conference on Mobile Systems, Applications, and Services (MobiSys'10). ACM, New York, NY, 21-34. DOI :http://dx.doi.org/10.1145/1814433.1814438

Ting Liu, Christopher M. Sadler, Pei Zhang, and Margaret Martonosi. 2004. Implementing software on resource-constrained mobile sensors: Experiences with impala and zebranet. In Proceedings of the 2nd International Conference on Mobile Systems, Applications, and Services (MobiSys'04). ACM, New York, NY, 256-269. DOI : http://dx.doi.org/10.1145/990064.990095

Michael J. McGlynn and Steven A. Borbash. 2001. Birthday protocols for low energy deployment and flexible neighbor discovery in ad hoc wireless networks. In Proceedings of the 2nd ACM International Symposium on Mobile Ad Hoc Networking and Computing (MobiHoc'01). ACM, New York, NY, 137-145. DOI : http://dx.doi.org/10.1145/501431.501435

Emiliano Miluzzo, Michela Papandrea, Nicholas D. Lane, Andy M. Sarroff, Silvia Giordano, and Andrew T. Campbell. 2011. Tapping into the vibe of the city using VibN, a continuous sensing application for smartphones. In Proceedings of the 1st International Symposium on From Digital Footprints to Social and Community Intelligence (SCI'11). ACM, New York, NY, 13-18. DOI:http://dx.doi. org/10.1145/2030066.2030071

M. Mitzenmacher and U. Upfal. 2007. Probability and Computing. Cambridge University Press.

Nintendo. 2012. Nintendo 3DS—Streetpass. (2012). Retrieved October 26, 2015, from http://www.nintendo. com/3ds/hardware.

H. L. Ivan Niven and Herbert S. Zuckerman. 1991. An Introduction to the Theory of Numbers. Wiley.

Jeongyeup Paek, Joongheon Kim, and Ramesh Govindan. 2010. Energy-efficient rate-adaptive GPS-based positioning for smartphones. In Proceedings of the 8th International Conference on Mobile Systems, Applications, and Services (MobiSys'10). ACM, New York, NY, 299-314. DOI : http://dx.doi.org/10. 1145/1814433.1814463

Anna-Kaisa Pietiläinen, Earl Oliver, Jason LeBrun, George Varghese, and Christophe Diot. 2009. MobiClique: Middleware for mobile social networking. In Proceedings of the 2nd ACM Workshop on Online Social Networks (WOSN'09). ACM, New York, NY, 49-54. DOI : http://dx.doi.org/10.1145/1592665.1592678

Aveek Purohit, Bodhi Priyantha, and Jie Liu. 2011. WiFlock: Collaborative group discovery and maintenance in mobile sensor networks. In Proceedings of the 2011 10th International Conference on Information Processing in Sensor Networks (IPSN'11). 37-48.

Jasmin Sasin. 2012. Shenzhen Ranks Fifth in the World in Terms of Population Density. Available at http://www.shenzhen-standard.com/2014/03/25/.

Softonic. 2012. Bluehoo Home Page. Retrieved October 26, 2015, from http://www.bluehoo.com.

Sony. 2013. PlayStation Home Page. Retrieved October 26, 2015, from http://us.playstation.com/psvita.

Synerge. 2013. Who's Near Me. Retrieved October 26, 2015, from http://www.windows7newsinfo.com/ smf/index.php?topic=9414.0; wap2.

Arvind Thiagarajan, James Biagioni, Tomas Gerlich, and Jakob Eriksson. 2010. Cooperative transit tracking using smart-phones. In Proceedings of the 8th ACM Conference on Embedded Networked Sensor Systems (SenSys'10). ACM, New York, NY, 85-98. DOI : http://dx.doi.org/10.1145/1869983.1869993

Yu-Chee Tseng, Chih-Shun Hsu, and Ten-Yueng Hsieh. 2002. Power-saving protocols for IEEE 802.11-based multi-hop ad hoc networks. In Proceedings of the Conference on Computer Communications (INFOCOM'02).

Wikipedia. 2013. Location-Based Game. Retrieved October 26, 2015, from http://en.wikipedia.org/wiki/ Location-based_game.

Tingxin Yan, Vikas Kumar, and Deepak Ganesan. 2010. CrowdSearch: Exploiting crowds for accurate realtime image search on mobile phones. In Proceedings of the 8th International Conference on Mobile Systems, Applications, and Services (MobiSys'10). ACM, New York, NY, 77-90.

Tingxin Yan, Matt Marzilli, Ryan Holmes, Deepak Ganesan, and Mark Corner. 2009. mCrowd: A platform for mobile crowdsourcing. In Proceedings of the 7th ACM Conference on Embedded Networked Sensor Systems (SenSys'09). ACM, New York, NY, 347-348. DOI : http://dx.doi.org/10.1145/1644038.1644094

Desheng Zhang and Tian He. 2012. pCruise: Reducing cruising miles for taxicab networks. In Proceedings of the Real-Time Systems Symposium (RTSS'12). 85-94. 
Desheng Zhang, Tian He, Yunhuai Liu, Yu Gu, Fan Ye, Raghu K. Ganti, and Hui Lei. 2012. Acc: Generic on-demand accelerations for neighbor discovery in mobile applications. In Proceedings of the 10th ACM Conference on Embedded Network Sensor Systems (SenSys'12). ACM, New York, NY, 169-182. DOI : http://dx.doi.org/10.1145/2426656.2426674

Rong Zheng, Jennifer C. Hou, and Lui Sha. 2003. Asynchronous wakeup for ad hoc networks. In Proceedings of the 4th ACM International Symposium on Mobile Ad Hoc Networking and Computing (MobiHoc'03). ACM, New York, NY, 35-45. DOI : http://dx.doi.org/10.1145/778415.778420

Received March 2014; revised April 2015; accepted September 2015 